\title{
Identification of specificity-defining amino acids of the wheat immune receptor Pm2 and powdery mildew effector AvrPm2
}

\author{
Manser, Beatrice ; Koller, Teresa ; Praz, Coraline Rosalie ; Roulin, Anne C ; Zbinden, Helen ; Arora,
} Sanu ; Steuernagel, Burkhard ; Wulff, Brande B H ; Keller, Beat ; Sánchez-Martín, Javier

\begin{abstract}
Plant nucleotide-binding leucine-rich repeat receptors (NLRs) act as intracellular sensors for pathogen-derived effector proteins and trigger an immune response, frequently resulting in the hypersensitive cell death response (HR) of the infected host cell. The wheat (Triticum aestivum) NLR Pm2 confers resistance against the fungal pathogen Blumeria graminis f. sp. tritici (Bgt) if the isolate contains the specific RNase-like effector AvrPm2. We identified and isolated seven new Pm2 alleles (Pm2e-i) in the wheat D-genome ancestor Aegilops tauschii and two new natural AvrPm2 haplotypes from Bgt. Upon transient co-expression in Nicotiana benthamiana, we observed a variant-specific HR of the Pm2 variants Pm2a and Pm2i towards AvrPm2 or its homolog from the AvrPm2 effector family, BgtE-5843, respectively. Through the introduction of naturally occurring non-synonymous single nucleotide polymorphisms and structure-guided mutations, we identified single amino acids in both the wheat NLR Pm2 and the fungal effector proteins AvrPm2 and BgtE-5843 responsible for the variant-specific HR of the Pm2 variants. Exchanging these amino acids led to a modified HR of the Pm2-AvrPm2 interaction and allowed the identification of the effector head epitope, a 20-amino-acid long unit of AvrPm2 involved in the HR. Swapping of the AvrPm2 head epitope to the non-HR-triggering AvrPm2 family member BgtE-5846 led to gain of a HR by Pm2a. Our study presents a molecular approach to identify crucial effector surface structures involved in the HR and demonstrates that natural and induced diversity in an immune receptor and its corresponding effectors can provide the basis for understanding and modifying NLR-effector specificity.
\end{abstract}

DOI: https://doi.org/10.1111/tpj.15214

Posted at the Zurich Open Repository and Archive, University of Zurich

ZORA URL: https://doi.org/10.5167/uzh-203367

Journal Article

Accepted Version

Originally published at:

Manser, Beatrice; Koller, Teresa; Praz, Coraline Rosalie; Roulin, Anne C; Zbinden, Helen; Arora, Sanu; Steuernagel, Burkhard; Wulff, Brande B H; Keller, Beat; Sánchez-Martín, Javier (2021). Identification of specificity-defining amino acids of the wheat immune receptor Pm2 and powdery mildew effector AvrPm2. The Plant Journal, 106(4):993-1007.

DOI: https://doi.org/10.1111/tpj.15214 
MS. BEATRICE MANSER (Orcid ID : 0000-0002-7592-5089)

Article type : Original Article

Identification of specificity-defining amino acids of the wheat immune receptor Pm2 and powdery mildew effector AvrPm2

Beatrice Manser ${ }^{1}$, Teresa Koller ${ }^{1}$, Coraline R. Praz ${ }^{1}$, Anne Roulin ${ }^{1}$, Helen Zbinden ${ }^{1}$, Sanu Arora ${ }^{2}$, Burkhard Steuernagel ${ }^{2}$, Brande B.H. Wulff², Beat Keller ${ }^{1^{*}}$, Javier Sánchez-Martín ${ }^{1^{*}}$

${ }^{1}$ Department of Plant and Microbial Biology, University of Zurich, Zollikerstrasse 107, 8008 Zurich, Switzerland

${ }^{2}$ John Innes Centre, Norwich Research Park, Norwich, NR4 7UH, United Kingdom

Pm2-AvrPm2 specificity

Plant immunity, Plant-fungi interactions, Pathogen effectors, Nucleotide-binding, leucine-rich repeat (LRR) immune receptor (NLR), NLR-effector specificity, Triticum aestivum, Blumeria graminis f. sp. tritici,

*Corresponding authors:

Beat Keller, Zollikerstrasse 107, 8008 Zurich, Switzerland, bkeller@botinst.uzh.ch Javier Sánchez-Martín, Zollikerstrasse 107, 8008 Zurich, Switzerland, javier.sanchezmartin@botinst.uzh.ch

This article has been accepted for publication and undergone full peer review but has not been through the copyediting, typesetting, pagination and proofreading process, which may lead to differences between this version and the Version of Record. Please cite this article as doi: 10.1111/TPJ.15214

This article is protected by copyright. All rights reserved 


\section{Summary}

Plant nucleotide-binding, leucine-rich repeat receptors (NLRs) act as intracellular sensors for pathogenderived effector proteins and trigger an immune response, frequently resulting in hypersensitive cell death response (HR) of the infected host cell. The wheat (Triticum aestivum) NLR Pm2 confers resistance against the fungal pathogen Blumeria graminis f. sp. tritici (Bgt) if the isolate contains the specific RNaselike effector AvrPm2. We identified and isolated seven new Pm2 alleles $(P m 2 e-i)$ in the wheat D-genome ancestor Aegilops tauschii and two new, natural AvrPm2 haplotypes from Bgt. Upon transient coexpression in Nicotiana benthamiana, we observed a variant-specific HR of the Pm2 variants Pm2a and Pm2i towards AvrPm2 or its homolog from the AvrPm2 effector family, BgtE-5843, respectively. Through the introduction of naturally occurring non-synonymous SNPs and structure-guided mutations, we identified single amino acids, in both the wheat NLR Pm2 and the fungal effector proteins AvrPm2 and BgtE5843 responsible for the variant-specific HR of the Pm2 variants. Exchanging these amino acids led to modified HR of the Pm2-AvrPm2 interaction and allowed the identification of the effector head epitope, a 20 amino acid long unit of AvrPm2 involved in HR. Swapping of the AvrPm2 head epitope to the non HRtriggering AvrPm2 family member BgtE-5846 led to gain of HR by Pm2a. Our study presents a molecular approach to identify crucial effector surface structures involved in HR and demonstrates that natural and induced diversity in an immune receptor and its corresponding effectors can provide the basis for understanding and modifying NLR - effector specificity.

This article is protected by copyright. All rights reserved 


\section{Introduction}

Ascomycete powdery mildews are widespread, agronomically important fungal pathogens of a large number of economically relevant crops (Glawe, 2008). They share an obligate biotrophic lifestyle as they are dependent on living host tissue for growth and reproduction. The powdery mildew disease of wheat (Triticum aestivum) is caused by the specialized, host-specific species of Blumeria graminis, the $B$. graminis forma specialis tritici (Bgt) (Dean and Van Kahn, 2012). To develop and sustain a successful infection and to avoid host recognition, Bgt relies on an arsenal of effector proteins (Müller et al., 2019).

Yield losses caused by Bgt have been frequently prevented by the use of fungicides, which can have a negative impact on ecosystem function (Dormann et al., 2007) and can result in the evolution of fungicide resistance (Lucas et al., 2015). A possibly more sustainable approach to reduce losses is the breeding for disease-resistant wheat cultivars by the introgression of resistance $(R)$ genes (Tanksley and Nelson, 1996). $R$ genes typically encode nucleotide-binding leucine-rich repeat receptor (NLR) proteins composed of an $\mathrm{N}$-terminal coiled-coil (CC) or Toll/interleukin-1 receptor (TIR) domain, a central nucleotide-binding (NB) domain and a C-terminal leucine-rich repeat (LRR) domain (Duxbury et al., 2016; Jones et al., 2016). Whereas animal NLRs often detect conserved microbe- or damage-associated molecular patterns (MAMPs/DAMPS), plant NLRs mostly act as intracellular sensors for pathogen-derived effectors, designated avirulence effector proteins (AVRs) (Jones et al., 2016; Dodds and Rathjen, 2010). The recognition of an effector by its corresponding NLR protein frequently results in a local hypersensitive cell death response (HR) of the infected cell limiting the spread of fungal infection (Lo Presti et al., 2015).

The natural diversity of wheat and its wild relatives represents a highly valuable resource for the discovery of new $R$ genes and their alleles (Bhullar et al., 2009; Arora et al., 2019). One of the largest known allelic series of plant resistance genes is the allelic series of the barley resistance gene Mla. Mla confers racespecific resistance to barley powdery mildew (Blumeria graminis f.sp. hordei, Bgh) and its allelic series encodes 23 known receptor variants (Seeholzer et al., 2010). The largest known NLR allelic series in wheat is formed by the wheat powdery mildew $(P m)$ resistance gene $P m 3$, which includes 17 functional alleles (Bhullar et al., 2010; Bhullar et al., 2009; Srichumpa et al., 2005; Yahiaoui et al., 2004).

The wheat repertoire of $R$ genes can be complemented with additional or improved $R$ genes either by conventional breeding or biotechnology (Rodriguez-Moreno et al., 2017; Sánchez-Martín and Keller, 2019). NLR engineering for extended pathogen recognition specificities is highly desired in plant disease resistance breeding (Rodriguez-Moreno et al., 2017). For example, in the wheat NLR Pm3f and the rice NLR Pikp1, mutants with single amino acid substitutions show enhanced and expanded HR, respectively 
(Stirnweis et al., 2014; De La Concepcion et al., 2019). Swaps of domains or single LRRs between allelic NLR variants are often used to analyze their role of in the effector recognition. For example, domain swaps between Pm3b and Pm3c, Mla1 and Mla6, and allelic variants of the flax rust resistance genes led to the identification of NLR regions, mostly in the LRR domain involved in effector recognition and to new recognition specificities of chimeric receptors (Brunner et al., 2010; Ravensdale et al., 2012; Shen et al., 2003; Ellis et al., 2007).

The wheat powdery mildew resistance gene Pm2, here re-named $P m 2 a$, encodes an NLR with an Nterminal CC domain, an NB (NB-ARC-subtype) and a C-terminal LRR domain containing 25 repeats (Sánchez-Martín et al., 2016). In co-infiltration assays in the heterologous, transient expression system Nicotiana benthamiana, the co-expression of the Pm2 and AvrPm2 results in a strong HR (Praz et al., 2017). The virulent AvrPm2 allele arose from a $12 \mathrm{~kb}$ deletion in the AvrPm2 locus on chromosome seven of the Bgt genome. Therefore, very little haplotype diversity of AvrPm2 has been found in the gene pool of Bgt (Praz et al., 2017; Müller et al., 2019). AvrPm2 is a member of a large gene family of cereal powdery mildew effectors consisting of 31 members: 17 members are from Bgt and 14 are from Bgh, respectively (Müller et al., 2019; Praz et al., 2017). They all encode RNase-like effector proteins, showing predicted structural homologies to the Ribonuclease T1 from Aspergillus phoenicis (Pedersen et al., 2012; Pace et al., 1991). The 3D structure of BEC1054 (CSEP0064) (Pennington et al., 2016; Pliego et al., 2013; Pedersen et al., 2012), a Bgh AvrPm2 family member, was solved by X-ray crystallization and confirmed its RNaselike structure (Pennington et al., 2019). Avra13, another Bgh family member, was shown to directly interact with the barley NLR Mla13 in yeast and in planta (Saur et al., 2019). AvrPm2 family members from Bgt include BgtE-5843 and BgtE-5846, the latter being the closest relative of AvrPm2 (Wicker et al., 2013). BgtE-5843 triggers HR upon co-expression with Pm2a in N. benthamiana which is not the case for BgtE-5846 (Praz et al., 2017; Praz et al., 2019). BgtE-5843 and BgtE-5846 are located together with BgtE5842 in a cluster of effector gene homologs at the AvrPm2 locus but are not affected by the $12 \mathrm{~kb}$ deletion affecting AvrPm2 (Praz et al., 2017). The gene expression varies drastically between the genes of this cluster. AvrPm2 is strongly expressed, whereas expression of BgtE-5843 and BgtE-5846 is not detected at $48 \mathrm{~h}$ post-infection (Praz et al., 2017; Müller et al., 2019).

Here, we first describe the identification and characterization of seven new Pm2 alleles from the wheat D genome ancestor Aegilops tauschii as well as the identification of two new, natural AvrPm2 haplotypes from Bgt. We detected a variant-specific HR of the Pm2 variants Pm2a and Pm2i towards AvrPm2 and BgtE-5843, respectively. Based on the introduction of naturally occurring non-synonymous SNPs and structure-guided mutations, we unraveled the molecular determinants responsible for the variant-specific

This article is protected by copyright. All rights reserved 
HR in both, the wheat NLR and the fungal Avr proteins. Amino acid changes in both, Pm2 and AvrPm2 modified NLR-effector specificity. Thus, we present an approach to identify the specific amino acids involved in NLR-AVR interactions using natural and induced diversity.

\section{Results}

\section{Isolation of novel Pm2 alleles from Ae. tauschii}

The Pm2-haplotype marker (Table S1) indicated the presence of the Pm2 gene in 48 of 487 tested bread wheat genotypes and 28 of 150 Ae. tauschii accessions (Table S2). Full-length sequencing of the Pm2 locus from the 76 putative Pm2-carrying accessions resulted in eight different Pm2 alleles (Table S3). Interestingly, the Pm2a allele was the only allele present in bread wheat, mostly of European origin (Table S2). This finding is in agreement with previous studies (Chen et al., 2019), suggesting that the Pm2a allele was the only allele introgressed into the bread wheat gene pool. The seven new Pm2 alleles were only identified in the Ae. tauschii accessions whereas Pm2a was absent among all tested Ae. tauschii accessions. The Pm2b (Ma et al., 2015) and Pm2c (Xu et al., 2015) alleles have been genetically described in the literature, although a recent publication reported that $P m 2 b$ and $P m 2 c$ are identical to $P m 2 a$ (Chen et al., 2019). Thus, we named the new Pm2 alleles Pm2d, Pm2e, Pm2f, Pm2g, Pm2h, Pm2i and Pm2j (Table S3). Although the original Pm2a allele was reported to not show alternative splicing variants, we further checked for the presence of alternative Pm2 transcripts in the newly identified Pm2 alleles by RT-PCR amplifying a fragment between the first exon and the $3^{\prime}$ UTR. This confirmed that all alleles led exclusively to the spliced product containing all three exons, encoding proteins of 1,276 or 1,277 amino acids in length (Table S3).

\section{Strong positive selection on LRR 21 of Pm2}

Given the absence of alternative splicing variants and the rare or absent polymorphisms in the $5^{\prime}$ UTR and $3^{\prime}$ UTR, sequence diversity and further analyses were limited to the gene-coding sequence (CDS), using the Pm2a allele as a reference. The new alleles contain single SNPs, deletions and/or combinations of shared SNPs (Table S4). The amino acid sequence alignment of the Pm2 protein variants (Figure 1) shows that Pm2e, Pm2f, Pm2i and Pm2j display high sequence similarity with two, six, three and four amino acid polymorphisms, respectively, compared to Pm2a. Pm2d, Pm2g and Pm2h are the most diverse proteins, with 15, 28 and 48 amino acid changes, respectively, occurring mostly in the LRR domain. Interestingly, an amino acid change at position 1167 (LRR domain) occurs in six out of the seven new Pm2 variants, resulting in the presence of four different amino acids at this position (Figure 1). The ratio between nonsynonymous (Ka) and synonymous substitutions (Ks) was determined for the complete coding sequence 
as well as for the different domains individually (CC, NB-ARC and LRR). Strong evidence for positive selection $(\mathrm{Ka} / \mathrm{Ks}=2.3)$ was observed in the gene region coding for the LRR domain (Table S5). The highly polymorphic position 1167 in LRR 21 is mainly responsible for the strong positive selection signal in the LRR domain. To visualize LRR 21 (1163-1186) and the amino acid at position 1167, we created a 3D-model of the LRR domain of Pm2 and concluded that position 1167 is located in the inner concave side of LRR 21 and is solvent-exposed (Figure S1).

\section{The Pm2 variants Pm2a and Pm2i show a variant-specific HR towards AvrPm2 and BgtE-5843 in} transient $\boldsymbol{N}$. benthamiana assays

We tested if AvrPm2 and its four closest Bgt AvrPm2 family members BgtE-5842, BgtE-5843, BgtE-5846 and BgtE-5901 (Wicker et al., 2013; Praz et al., 2017) trigger HR when co-infiltrated with the seven novel $\mathrm{Pm} 2$ variants and Pm2a in the heterologous $N$. benthamiana system. Six of the eight Pm2 variants elicited different levels of HR when co-infiltrated with AvrPm2 (Figure 2a, 2c and Figure S2). Pm2a triggered the strongest HR upon co-infiltration with AvrPm2, followed by Pm2e, Pm2i, Pm2j and Pm2f. Pm2g elicited a very weak HR, whereas Pm2d and Pm2h did not trigger HR upon AvrPm2 co-infiltration. There was an inverse relation between the intensity of $\mathrm{HR}$ upon co-infiltration with AvrPm2 and the number of polymorphisms of the new Pm2 variants compared to Pm2a (Figure 2a, 2c , Figure S3 and Table S3). The strong HR elicited by Pm2a and Pm2e in presence of AvrPm2 were not significantly different and suggest that Pm2a and Pm2e are the optimal variants among the tested ones for triggering HR when co-infiltrated with AvrPm2.

Pm2a, Pm2e, Pm2f, Pm2i and Pm2j elicit HR when co-infiltrated with BgtE-5843 (Figure 2b, 2c and Figure S2). In this case, Pm2i gave the strongest response. Pm2i gave a significantly stronger HR upon coinfiltration with BgtE-5843 compared to Pm2a, Pm2e and Pm2f, even though the response was lower in amplitude compared to the Pm2a-AvrPm2 response (Figure 2b). Pm2d, Pm2g and Pm2h did not elicit HR upon co-infiltration with BgtE-5843. BgtE-5846 and BgtE-5842 were not did not trigger HR with any of the eight Pm2 variants (Figure S2). BgtE-5901 gave no HR with any of the Pm2 variants, although in coinfiltrations with Pm2i, BgtE-5901 showed a statistically significant difference to BgtE-5846 (Figure S2 and Table S6). However, the HR of BgtE-5901 in co-infiltrations with Pm2i was extremely weak. Western blot analysis confirmed protein accumulation of all AvrPm2 family members and Pm2 variants (Figure 2d). Pm2d showed a lower protein accumulation than the other Pm2 variants, which could be the reason for Pm2d not eliciting HR. For the other Pm2 variants, the level of HR response was not correlated to the level of protein accumulation (Figure 2). The strongest HR was observed upon co-infiltration of Pm2a with 
AvrPm2 and Pm2i with BgtE-5843, suggesting a variant-specific HR of Pm2a and Pm2i towards AvrPm2 and BgtE-5843, respectively.

\section{Amino acid position 1167 of Pm2 defines the variant-specific HR of Pm2a and Pm2i}

Pm2a, Pm2e and Pm2i only differ at three amino acid positions: Pm2 ${ }^{170}, \mathrm{Pm}^{398}$ and Pm2 ${ }^{1167}$ (Figure 1). Pm2a and Pm2e share a glycine at position 1167 and both trigger higher levels of HR upon co-infiltration with AvrPm2 compared to Pm2i. The latter differs from Pm2e and Pm2a at position 1167 and gives a stronger HR upon co-infiltration with BgtE-5843 compared to Pm2a and Pm2e (Figure 2b, 2c). These results indicate that amino acid 1167 plays a major role in the Pm2 variant-specific response to AvrPm2 and BgtE-5843. To further investigate the molecular basis of the variant-specific HR of Pm2a and Pm2i, we performed site-directed mutagenesis. We focused on the three amino acid polymorphisms between Pm2a and Pm2i and co-infiltrated the C-terminal HA-tagged mutants with all possible amino acid combinations with AvrPm2 and BgtE-5843, respectively. Pm2a mutants at position 170 and 398 with an exchange to the amino acids of Pm2i (Pm2a $\left.a^{\mathrm{S170N}}, \mathrm{Pm} 2 \mathrm{a}^{\mathrm{L398V}}, \mathrm{Pm} 2 \mathrm{a}^{\mathrm{S170N} / \mathrm{L398V}}=\mathrm{Pm} 2 \mathrm{e}\right)$ showed similarly strong HR as Pm2a when co-infiltrated with AvrPm2 (Figure 3a). When co-infiltrated with BgtE-5843, the same mutants showed decreased HR when compared to Pm2a (Figure $3 \mathrm{~b}$ ), indicating that positions $\mathrm{Pm} 2 \mathrm{a}^{\mathrm{S170}}$ and Pm2a $a^{L 398}$ are important for HR in response to BgtE-5843 but not to AvrPm2. All Pm2a mutants containing the exchange of glycine at position 1167 to the aspartic acid of Pm2i (Pm2aG1167D, Pm2a S170N/G1167D, Pm2a ${ }^{\text {L398V/G1167D }}$ ) showed a significantly reduced HR compared to Pm2a upon co-infiltration with AvrPm2 (Figure 3a,c). When the Pm2a mutants at position 1167 were co-infiltrated with BgtE-5843, they showed an intermediate HR, similar to Pm2a or Pm2i (Figure 3b). These results indicate that Pm2a ${ }^{G 1167}$ and Pm2i ${ }^{D 1167}$ are important for the variant-specific HR triggered by AvrPm2 and BgtE-5843, respectively. Furthermore, positions 170 and 398 are important for HR in response to BgtE-5843 in Pm2a, but not in Pm2i.

Taken together, the results of the site-directed mutagenesis study support our hypothesis that position 1167 in the Pm2 protein determines the variant-specific HR of Pm2a and Pm2i towards AvrPm2 and BgtE5843, respectively. Pm2a triggers the strongest HR in co-infiltrations with AvrPm2, and when amino acid 1167 in Pm2a is changed from glycine to aspartic acid this preference disappears. Although the differences in HR levels of Pm2a and Pm2i in co-infiltrations with BgtE-5843 are subtle, this preference was consistently observed in co-infiltration with different effector : NLR ratios (Figure S4).

In contrast to the glycine in Pm2a, of which the residue consists solely of a hydrogen atom, the aspartic acid side chain in Pm2i is predicted to protrude prominently in the 3D-model (Figure 3d,e). When a glycine

This article is protected by copyright. All rights reserved 
replaces aspartic acid in Pm2i $\left(\mathrm{Pm}^{\mathrm{a}} \mathrm{a}^{\mathrm{S170N/L398V}}=\mathrm{Pm} 2 \mathrm{i}^{\mathrm{D} 1167 G}\right.$ ) (Figure 3a,c), an HR comparable to Pm2a is elicited upon co-infiltration with AvrPm2. On the other hand, Pm2a with the aspartic acid instead of the glycine shows a significantly reduced HR when co-infiltrated with AvrPm2 (Figure 3a,c). We suggest a model where the aspartic acid in Pm2i is a steric hindrance for the interaction with AvrPm2 but plays an important role in the interaction with BgtE-5843 since Pm2i mutants containing amino acid exchanges at position 1167 show a significantly lower HR when co-infiltrated with BgtE-5843. In this model, the glycine in Pm2a does not interfere in the interaction with AvrPm2. To conclude, amino acid 1167 seems not to be involved in HR in response to AvrPm2 by Pm2a, however, it seems to be involved in the response to BgtE5843 by Pm2i.

\section{AvrPm2-H1, one of two new AvrPm2 variants triggers HR in co-infiltrations with Pm2a}

In addition to the study on the Pm2 alleles, we also analyzed AvrPm2 diversity in our in-house Bgtcollection and discovered that the Bgt isolates USA7 and USA2 each harbours a new AvrPm2 haplotype (H); AvrPm2-H1 (USA7) and AvrPm2-H2 (USA2). The amino acid sequence alignment of the AvrPm2 variants using AvrPm2 as a reference (Praz et al., 2017) revealed that AvrPm2-H1 differs from AvrPm2 at position 67 (G67S) (Figure 4a). This polymorphism is shared by AvrPm2-H2, which has an additional polymorphism at position 96 (V96D).

In a leaf segment infection assay we tested the Bgt USA7 and USA2 isolates on near isogenic wheat lines carrying the Pm2a allele (Figure S5). The AvrPm2-H1 carrying USA7 isolate was avirulent on the Pm2a near isogenic lines. In contrast, the USA2 isolate carrying AvrPm2-H2 showed a virulent phenotype, suggesting that AvrPm2-H1 but not AvrPm2-H2 is recognized by Pm2a.

Co-infiltrations of the AvrPm2-H1 and AvrPm2-H2 variants with Pm2a in N. benthamiana demonstrated that AvrPm2-H1 triggers a HR of similar intensity as AvrPm2 (Figure 4b). In contrast, AvrPm2-H2 did not trigger HR when co-infiltrated with Pm2a. This result is in agreement with the observations from the leaf segment infection tests (Figure S5). To verify if the polymorphism at position 96 (V96D) alone is responsible for the abolished HR of AvrPm2-H2, we exchanged in AvrPm2 valine at position 96 with aspartic acid (construct E103, Table S7). In co-infiltrations of Pm2a and E103 no HR was observed, suggesting that V96 of AvrPm2 is important for HR induction. Protein analysis by western blot demonstrated the presence of the tested AvrPm2 variants (Figure 4c).

\section{Modeling of AvrPm2 and BgtE-5846 demonstrates high structural similarities}

From the AvrPm2 variant analyses we conclude that amino acid V96 of AvrPm2 is crucial for HR in coinfiltrations with Pm2a. We wanted to further determine the effector amino acids which are crucial for 
HR. We chose to use effector BgtE-5846, which has $55.2 \%$ amino acid sequence similarity compared to AvrPm2 as a source of diversity information. BgtE-5846 is the closest related family member of AvrPm2 (Wicker et al., 2013) but does not trigger a response with any of the Pm2 variants (Figure S2). In contrast, BgtE-5843 is more distantly related to AvrPm2 (35.4\% sequence similarity), and despite the lower sequence similarity it triggers HR in co-infiltrations with five Pm2 variants (Figure $2 b$ ). We created a sequence alignment of AvrPm2, BgtE-5843 and BgtE-5846 (Figure 5a). Position 96 is a valine in all three effectors. In fact, the C-terminal end of non HR-triggering effector Bgt-E5846 and HR-triggering effector Bgt-E5843 are more similar to each other than to the C-terminal end of AvrPm2. We used the structure of the Bgh effector BEC1054 (PDB ID: 6FMB) (Pennington et al., 2019) as template to create high confidence 3D models of AvrPm2 and BgtE-5846. Superposition of the two 3D models demonstrated high predicted structural similarity despite the relatively low $55.2 \%$ sequence similarity (Figure $5 b$ ).

\section{A "head epitope" of AvrPm2 is critical for HR induction by Pm2a}

Using the 3D models of AvrPm2 and BgtE-5846 we wanted to investigate which part of AvrPm2 is crucial for HR. The 3D models of AvrPm2 and BgtE-5846 were used to divide the proteins into eight units, ranging from six to 20 amino acids (Figure 5a,c). The corresponding parts of AvrPm2 and BgtE-5846 all have the same length, except for the part containing the C-terminus, which is one amino acid shorter in BgtE-5846. By swapping these units between AvrPm2 and BgtE-5846 we aimed to identify an AvrPm2 protein surface responsible for HR in co-infiltrations with $\mathrm{Pm} 2 \mathrm{a}$, which would lead to a gain of HR when transferred to BgtE-5846 and a loss of HR when AvrPm2 surface was replaced by amino acids of BgtE-5846. Each of the eight units was swapped bidirectionally between AvrPm2 and BgtE-5846 resulting in 16 constructs (E25E40, Table S7), eight of them were AvrPm2 mutants carrying single unit of BgtE-5846 and vice versa. The constructs were co-infiltrated with Pm2a in N. benthamiana. Swapping seven units between AvrPm2 and BgtE-5846 did not change the HR compared to the corresponding wild-type effectors AvrPm2 and BgtE5846, respectively, indicating no involvement of these seven units in HR. However, constructs E25 and E33 (Figure 6a) exhibited a different HR than their corresponding wild-type effectors AvrPm2 and BgtE-5846. The E25 construct, with the unit \#1 of BgtE-5846 in the background of the AvrPm2 protein, did no elicit HR (Figure 6b). Surprisingly, the corresponding reverse swap, construct E33 with the unit \#1 of AvrPm2 in the background of the BgtE-5846 protein, resulted in gain of HR (Figure 6b). This unit \#1, which consists of 13 amino acids at the $\mathrm{N}$-terminal part and seven amino acids at them $\mathrm{C}$-terminal part of the effector (colored red in Figure 5a,c), will be further referred to as the "head epitope". We concluded that the head epitope of AvrPm2 is the protein surface involved in Pm2a-mediated HR. However, the HR elicited by E33 was significantly weaker compared to the one of AvrPm2 (Figure 6b), indicating that not only the head epitope

This article is protected by copyright. All rights reserved 
but also the rest of AvrPm2 is needed for a wild-type level of HR. Protein analysis by western blot demonstrated the presence of proteins E25 and E33 (Figure 6c).

\section{The C-terminal part of the AvrPm2 head epitope is essential for Pm2a-mediated HR}

The wild type head epitopes of AvrPm2 and BgtE-5846 consist of 20 and 19 amino acids, respectively and differ by eight amino acids: four (SA3, D/N6, K/N8 and I/T10) are located in the N-terminal part of the head epitope and four (T/D94, L/A97, R/G98 and D/Stop99) are located in the C-terminal part (Figure 5a and Figure S6). To further characterize the role of the head epitope in the Pm2a-mediated HR, the N- and C- terminal parts were swapped bidirectionally between AvrPm2 and BgtE-5846 (constructs E82-E85, Table S7) (Figure 6a). AvrPm2 with the N-terminal part of BgtE-5846 (E84) elicited a strong HR, slightly weaker compared to the wild type AvrPm2 (Figure 6b). The swap of the N-terminal part of AvrPm2 to BgtE-5846 (E82) did not lead to gain of HR (Figure 6b). Based on these results, we conclude that the Nterminal part of the AvrPm2 head epitope alone is not sufficient to elicit HR when transferred to BgtE5846, but might quantitatively contribute to the HR induction by Pm2a. Interestingly, the swap of the Cterminal part of BgtE-5846 to AvrPm2 (E85) completely abolished HR, whereas BgtE-5846 with the Cterminal part of AvrPm2 (E83) elicited weak HR (Figure 6b). An additional construct with a deletion of the C-terminal part of the head epitope of AvrPm2 was created (E64), which showed a complete loss of HR (Figure 6b). These results, together with the results from the AvrPm2 haplotype analysis indicate that the C-terminal part of the AvrPm2 head epitope is involved in Pm2a-mediated HR. Total protein extraction followed by western blot demonstrated the protein presence of the tested constructs (Figure $6 \mathrm{c}$ ). There was no obvious correlation of protein accumulation with the level of HR.

To further determine which of the eight amino acid polymorphisms in the head epitope are needed for HR induction by $\mathrm{Pm} 2$, we created 18 new constructs. In eight constructs, eight amino acids in the head epitope of BgtE-5846 were individually replaced by the ones of AvrPm2 at this position (E74-E81, Table S7), including construct E78. Construct E78 contains T94 from AvrPm2, which is the only amino acid in the head epitope which is the same in AvrPm2 as in the HR-triggering BgtE-5843, but different in the non HRtriggering BgtE-5846 (Figure 5a). None of the single amino acid exchanges, including construct E78 (Figure $6 d)$, led to elicitation of HR. The remaining ten constructs contained combined amino acid exchanges in the head epitope of BgtE-5846 with amino acids of AvrPm2 (E88-E97, Table S7). Constructs E88 and E97 (Figure 6a), with such combined exchanges, triggered a weak, but significantly stronger HR compared to BgtE-5846 (Figure 6d). However, construct E97 was not detectable on western blot, thus the weak HR response could be due to very low protein accumulation (Figure 6c). The construct E88 consists of exchanges A3S, A97L, G98R and STOP99D in BgtE-5846 and construct E97 consists A3S, T10S, D94T and

This article is protected by copyright. All rights reserved 
A97L in BgtE-5846, respectively (Figure 6a). The amino acid exchanges A3S and A97L shared between constructs E88 and E97, led to a gain of weak HR, suggesting that position 3 and 97 of AvrPm2 are likely involved in HR mediated by Pm2a. These results are supported by the decreased HR triggered by construct E43, an AvrPm2 mutant consisting of S3A, F14Y and L97A (Figure 6a,d and S7 Table). However, the two amino acids at position 3 and 97 alone are not sufficient to elicit a wild type-like HR in BgtE-5846 and in case of E97 could be involved in protein stability. Protein detection by western blot demonstrated the presence of all constructs, except E97 (Figure 6c).

\section{Head epitopes of BgtE-5843 and AvrPm2 are key structures in Pm2 variant-specific HR}

To check if the head epitope of AvrPm2 is also involved in HR triggered by other Pm2 variants, we coinfiltrated E33 (Figure 6a and Figure 7a) with the seven new Pm2 variants and Pm2a. In addition to Pm2a, Pm2e triggered an HR as well (Figure 7b). Further, we were interested if the head epitope of BgtE-5843 is also the protein surface involved in Pm2-mediated HR. Therefore, we swapped the head epitope of BgtE5843 to BgtE-5846 (E98) (Figure 7a) and co-infiltrated it with the seven new Pm2 variants and Pm2a. Pm2i elicited a weak HR upon co-infiltration with E98 (Figure 7c). This further supports our hypothesis of a Pm2 variant-specific HR, where Pm2a and Pm2e specifically trigger the strongest HR in co-infiltrations with AvrPm2 and in co-infiltrations with BgtE-5843 the Pm2i-mediated HR is the strongest, with the head epitope as the key unit involved in HR and specificity. The presence of the E98 protein was demonstrated on the western blot (Figure 6c).

\section{Discussion}

\section{Sequence diversity at the Pm2 locus in the wheat and Ae. tauschii gene pool}

Here we isolated seven new Pm2 alleles from the wheat D genome ancestor Ae. tauschii, constituting together with Pm2a a new allelic series of a Pm gene. Six Pm2 variants trigger HR when co-infiltrated with AvrPm2 in $N$. benthamiana. This demonstrates the relevance of wild wheat relatives as reservoirs of functional $R$ genes and their alleles to broaden the resistance gene pool of elite wheat cultivars. Other well studied allelic series of resistance genes include Pm3 (Bhullar et al., 2010; Bhullar et al., 2009; Srichumpa et al., 2005; Yahiaoui et al., 2004), the barley Mla allelic series (Seeholzer et al., 2010) and the flax rust resistance locus $L$ with 13 functional $L$ alleles (Ellis et al., 1999). The Pm2 allelic series shows a very high sequence identity (>97.6\%), slightly higher compared to the Pm3 series (>97\%) (Bhullar et al., 2010) and higher compared to the $L$ and Mla allelic series (Seeholzer et al., 2010; Ellis et al., 1999), indicating a recent divergence of the $P m 2$ alleles. A high Ka/Ks ratio revealed a strong positive selection acting on the LRR domain, mainly caused by the amino acid diversity at position 1167 , indicating a major 
role of the LRR domain in the effector specificity. This was also found in the LRR domains of the Pm3 and Mla genes (Bhullar et al., 2010; Seeholzer et al., 2010). The study of Pm2a and Pm2i mutants in N. benthamiana indicated that the glycine at position 1167 in Pm2a and Pm2e is responsible for the strong HR upon AvrPm2 co-infiltration. Given the natural diversity as well as the functional importance of position 1167, it might be worth testing the amino acids at this position occurring in other Pm2 variants for function in resistance.

\section{Variant-specific HR of Pm2 variants towards AvrPm2 and BgtE-5846 and NLR modification}

In this study, we characterized the molecular basis of the variant-specific HR of Pm2a and Pm2i towards AvrPm2 and BgtE-5843, respectively, and demonstrate that this preference can be modified by exchanging amino acids between individual Pm2 variants. Although the design of NLRs for extended pathogen specificities is highly desired in plant disease resistance breeding (Rodriguez-Moreno et al., 2017), only a few studies describe modified NLRs with increased or novel effector recognition spectra. Stirnweis et al. substituted two amino acids in the ARC2 subdomain of the wheat NLR Pm3f, which are crucial for a fast and strong HR and enhanced the capacity of Pm3f to induce HR in N. benthamiana and enlarged the resistance spectrum in wheat (Stirnweis et al., 2014). In contrast, here we modified the effector specificity of Pm2 through the exchange of one specific amino acid in the C-terminal end of the LRR domain. Pm2i mutants with the exchange of the aspartic acid at position 1167 by a glycine (Pm2jD11676 $=P m 2 e, P m 2 i^{N 170 S / D 1167 G}=P m 2 a^{L 398 V}$ and $P m 2 i^{V 398 L / D 1167 G}=P m 2 a^{S 170 N}$ ) showed a Pm2a-like HR response when co-infiltrated with AvrPm2. Several studies based on LRR swap experiments demonstrated that pathogen recognition specificity of plant NLRs is determined by their LRR domains (Shen et al., 2003; Brunner et al., 2010; Ellis et al., 2007). The specificity can be controlled by the C-terminal LRRs alone (Shen et al., 2003) or may also involve the N-terminal LRRs (Brunner et al., 2010). Pm3 is an example for both cases; for Pm3a-specific resistance, the C-terminal LRR motifs are required but for Pm3b- and Pm3cmediated resistance also N-terminal LRR motifs are essential (Brunner et al., 2010). For example, Pm3a with an amino acid exchange in the C-terminal LRR27, the Pm3a- $\delta 5$ mutant showed a lower activation threshold than the natural Pm3a and an expanded recognition spectrum towards naturally occurring AvrPm3 ${ }^{\mathrm{A} 2 / \mathrm{F2}}$ variants (Lindner et al., 2020). LRR swaps between the barley Mla1 and Mla6 (Shen et al., 2003; Bauer et al., 2021), as well as swaps between Mla10 and Mla22 (Bauer et al., 2021) demonstrated that the effector recognition specificities are determined by the C-terminal LRRs of the Mla proteins. LRR swaps between either the flax L6 and L11 or L5 and L6 variants revealed that the last four LRRs of L6 are required for the recognition of the flax rust effector AvrL567-D (Ravensdale et al., 2012; Ellis et al., 2007). Whereas the region conferring specificity of L6 and Pm3a show 13 amino acid polymorphisms compared 
to L5 and other Pm3 variants, respectively (Ravensdale et al., 2012; Brunner et al., 2010), we identified a single amino acid at the solvent-exposed position 1167 in LRR 21 that is crucial for specificity of Pm2.

Random-mutagenesis was often used to identify amino acids of NLRs involved in the recognition of their corresponding Avrs and to modify their specificity. Increased sensitivity and expansion of the specificity was achieved through random mutagenesis in the potato NLRs R3a and Rx, recognizing the Phytophthora infestans effector AVR3a (Armstrong et al., 2005; Bos et al., 2009) and the potato virus X elicitor CP-TK (Bendahmane et al., 1995), respectively. Sensitized mutants were achieved through substitutions in the NB-ARC domain of R3a (Harris et al., 2013) and Rx (Segretin et al., 2014), possibly due to a lower threshold for activation. In these two NLRs, substitutions in the LRR domain expanded the specificity towards the effector/elicitor variants AVR3a ${ }^{\mathrm{EM}}$ (R3a mutants) (Segretin et al., 2014) and CP-KR (Rx mutants) (Farnham and Baulcombe, 2006), respectively. Specificity was also modified and expanded in the NLR-effector pair Pik - AVR-Pik, where the integrated HMA domain the rice Pik-1 directly binds AVR-PikD from the rice blast pathogen Magnapothe oryzae (Kanzaki et al., 2012). Recently, structure-guided engineering of the PikpHMA domain lead to an expanded, Pikm-like HR of the Pikp ${ }^{N K-K E}$ variant to AVR-PikE and AVR-PikA in N. benthamiana, respectively (De La Concepcion et al., 2019). RGA5, another rice NLR with an integrated HMA, domain binds to its corresponding M. oryzae effectors via a different HMA binding interface (Guo et al., 2018) than Pik, suggesting that an HMA domain could be engineered to bind and respond to multiple M. oryzae effectors (De La Concepcion et al., 2019; Guo et al., 2018). A similar modification of Pm2 towards the additional detection of other Bgt effectors like AvrPm3 might be hindered by the fact that in Pm2 as well as in Pm3 the C-terminal LRR motifs are required for effector recognition (Brunner et al., 2010). Nevertheless, we demonstrated that the AvrPm2-induced HR can be modified through Pm2 $2^{1167}$ modification. To conclude, we were able to modify the HR elicited by Pm2 by the use of diversity within the Pm2 allelic series.

In this study, NLR-effector specificity was studied by quantitative measurement of cell death responses in transient co-expression experiments in the heterologous system $N$. benthamiana. The results obtainded in this system may not always reflect the interactions in the native wheat-powdery mildew system, in particular if additional components from the pathogens (e.g. modifiers) contribute to the interaction specificity. Nevertheless, in several other studies the NLR-effector specificities observed in $N$. benthamiana could be confirmed when the experiments were performed in the native plant system (Bourras et al., 2015; Bourras et al., 2019; Saur et al., 2019).

\section{Head-epitope of AvrPm2 and related effectors is involved in Pm2a-mediated HR}

This article is protected by copyright. All rights reserved 
In a structure-informed mutagenesis screen on AvrPm2 we identified a 20 amino acid long unit, the head epitope, determining specificity on the effector side in the NLR-AVR interaction between Pm2a and AvrPm2 and Pm2i and BgtE-5843, respectively. Thus, the identification of the head epitope and the newly discovered AvrPm2-H2 variant which does not trigger a Pm2a-mediated HR indicate the importance of the C-terminal end of AvrPm2 for HR mediated by Pm2. Previous effector mutagenesis studies in the literature mostly used natural polymorphisms among effector variants or families. For instance, a mutagenesis study on AvrL567 using amino acid polymorphisms across the allelic variants led to the identification of three amino acids involved in the recognition by the $L$ proteins and suggested that the specificity results from the additive effect of multiple contact points with the $L$ protein (Ravensdale et al., 2012; Dodds et al., 2006). Chimeric effectors between Avra10 and Avra22 demonstrated that four (Avra10) and five (Avra22) amino acids are necessary to confer Mla10- and Mla22-specific recognition, respectively (Bauer et al., 2021). These findings suggest, that multiple amino acids on the Avra effector surface determine the recognition-specificities of Mla proteins. In contrast, the head epitope is a small, rather defined region of AvrPm2 that is crucial for HR. The crystal structure of the Pikp-HMA/AVR-PikD complex allowed the design of AVR-PikD mutants using allelic polymorphisms, which showed an impaired Pikp binding in $\mathrm{Y} 2 \mathrm{H}$ and in rice. These results confirmed the recognition site of AVR-PikD, previously identified in the structure of the Pikp-HMA/AVR-PikD complex (Maqbool et al., 2015). Similar to AVR-PikD, where a crystal structure of the effector was available, we used the AvrPm2 3D-model to focus on solvent-exposed amino acids in the structure-informed mutagenesis screen. AvrPm3 ${ }^{\mathrm{Az} / \mathrm{F} 2}$, a Bgt effector, is recognized by Pm3a and Pm3f (Bourras et al., 2015). Through AvrPm3 ${ }^{\mathrm{A} 2 / \mathrm{F2}}$ mutants containing amino acid exchanges from the 24 AvrPm3 $3^{A 2 / F 2}$ family members, eight amino acids could be identified that individually led to an enhanced HR with either Pmf3a, Pm3f $\mathrm{f}^{\mathrm{L} 56 \mathrm{P} / \mathrm{Y} 458 \mathrm{H}}$ or both (McNally et al., 2018). Domain swap experiments between $\mathrm{AvrPm}^{\mathrm{B} 2 / \mathrm{C} 2}$ and the non-recognized Bgt-51460 revealed that two amino acid segments of $A v r P m 3^{\mathrm{B} 2 / \mathrm{C} 2}$ are sufficient to trigger $\mathrm{HR}$ in co-infiltration with $\mathrm{Pm} 3 \mathrm{~b}$ and $\mathrm{Pm} 3 \mathrm{c}$, respectively (Bourras et al., 2019). Similarly, regions of the non HR-triggering BgtE-5846 needed to be engineered to achieve a gain of HR phenotype, suggesting the involvement of several amino acids in the head epitope of AvrPm2 in the NLR-AVR interaction.

C-termini of other effectors were reported to have a similar essential role as the C-terminus of AvrPm2 does in the Pm2a-mediated HR. The 75-amino acid C-terminal region of AVR3a ${ }^{\mathrm{Kl}}$ was sufficient to trigger R3a-mediated HR in N. benthamiana. The deletion of 16 amino acids within this region led to loss of R3amediated HR, as well as to loss of the effector virulence function (Bos et al., 2006). C-terminus truncations of the flax rust AvrM-A led to a loss of flax M-dependent HR (Catanzariti et al., 2010).

This article is protected by copyright. All rights reserved 
Despite the low amino acid sequence similarity between the homologous RNase-like effectors AvrPm2 and BgtE-5843, the same structural surface is crucial for HR mediated by different Pm2 variants. AvrPm2 and BgtE-5843 belong to a class of RNase-like effectors with a single, strictly conserved intron (Spanu, 2017), and it is likely that this class accounts for about $10-15 \%$ of all predicted candidate effector proteins in cereal powdery mildews (Pedersen et al., 2012; Menardo et al., 2017). Additionally, it is known that effector families of cereal powdery mildews show high sequence variation, while the predicted structures appear to be conserved (Bourras et al., 2016; Franceschetti et al., 2017). Therefore, we hypothesize that also other RNase-like effectors from diverse cereal powdery mildews are recognized through their head epitopes by the corresponding immune receptors. Another RNase-like effector and an AvrPm2 family member is Avra13, which was recently shown to interact directly in yeast and planta with the corresponding barley receptor Mla13 (Saur et al., 2019), although the recognition site of Avra13 by Mla13 has not been identified. Because protein-engineering of both, AvrPm2 family members and Pm2 variants resulted in an altered HR, a direct protein-protein interaction of AvrPm2 and Pm2 seems likely. However, considering the recent discovery of the wheel-like pentameric ZAR1 resistosome, a multi-component system which is needed for activation of the Arabidopsis thaliana NLR ZAR1 (Wang et al., 2019), yet unknown factors could be involved in the Pm2-AvrPm2 interaction.

\section{Experimental procedures}

\section{Plant material, growth conditions, DNA isolation and infection tests}

A collection of 487 wheat accessions (Pont et al., 2019) and 250 Ae. tauschii accessions (S8 Table), consisting of 150 Ae. tauschii ssp. strangulate accessions (Arora et al., 2019) and 100 Ae. tauschii accessions of our in-house collection, was screened for the presence of Pm2, using a Pm2 haplotypespecific marker (S1 Table) (Sánchez-Martín et al., 2016). DNA isolation was performed as previously described (Stein et al., 2001). Leaf segment infection test with powdery mildew was done as previously described (Sánchez-Martín et al., 2016). The AvrPm2 haplotype carrying isolates USA2 and USA7 were tested on the near isogenic lines Ulka/8*Chancellor, Federation*4/Ulka and CI12632/8*Chancellor carrying the Pm2a allele (Parlange et al., 2015; Sánchez-Martín et al., 2016).

\section{Isolation of new Pm2 alleles}

After the identification of Pm2-carrying accessions, a long range PCR was performed, followed by a nested PCR reaction. The PCR products were sequenced with internal primers (S1 Table) as previously described (Sánchez-Martín et al., 2016). Sequence assembly, consensus development, alignments and the extraction 
of the protein sequence were done using CLC Genomics Workbench, version 8.1 (Qiagen Bioinformatics, Aarhus, Denmark).

\section{Constructs for co-expression assay in $\mathbf{N}$. benthamiana}

Pm2 alleles were amplified by PCR from Ae. tauschii DNA and were cloned into Gateway system compatible entry vectors via Gateway BP Clonase II reactions (ThermoFisher Scientific, Waltham, Massachusetts, USA). Introduction of modifications was done by site-directed mutagenesis. Effector constructs without signal peptide and flanking Gateway compatible cloning sites resulting in a Gateway compatible cloning vector (pUC57) were synthesized by BioCat (https://www.biocat.com/). The desired construct was then directly cloned into expression vector pIPKb004 (Himmelbach et al., 2007) carrying a 35 s promoter using LR Clonase II (ThermoFisher Scientific). Sequence integrity was confirmed by Sanger sequencing.

\section{Transient protein expression assays in $N$. benthamiana}

Agrobacterium tumefaciens strain GV3101 was transformed with the desired constructs by the freezethaw protocol (Hofgen and Willmitzer, 1988). Transient expression by agroinfiltration in $N$. benthamiana was performed as previously described (Bourras et al., 2019). Cultures were mixed in ratio 1:4 (NLR:effector) and infiltrated into leaves of 13 day old $N$. benthamiana plants.

HR was assessed two days post-infiltration by fluorescence scanning as previously described (Praz et al., 2017) of the abaxial side of the leaf and HR was quantified with the ImageJ image analysis software measuring the mean grey value of the infiltrated area. Since we compared the results of two combinations with each other and the data was not always normally distributed, we performed a twosample Wilcoxon rank sum test. Statistical significance is indicated. For HR quantification two independent experiments, each consisting of 7-8 independent leaf replicates were used. An exception is the co-infiltration of Pm2i with BgtE-5843 from Figure 3a, where only one experiment was performed.

\section{Plant protein extraction and western blot analyses}

Tissue for western blot analyses was harvested two days post infiltration and immediately frozen using liquid nitrogen. Leaf material was ground and heated in SDS sample buffer. Samples were separated on SDS-PAGE gels and transferred to nitrocellulose membranes (GE Healthcare, Chicago, Illinois, USA). Membranes were probed with monoclonal HRP-conjugated anti-HA antibody (Roche, Basel, Switzerland). The detection of the antibodies was performed with WesternBright ECL HRP substrate (Advansta, San Jose, California, USA) and the use of the Fusion FX system.

This article is protected by copyright. All rights reserved 


\section{D-protein structure models}

The 3D-protein structure of the LRR domains of Pm2 a and Pm2i was created by the RaptorX protein prediction program (http://raptorx.uchicago.edu) using the crystal structure of Toll-like receptor 13 (PDB ID: 4ZOC) as best template with a p-value of 5.24e-22 (Pm2a )and 1.09e-21(Pm2i). For the 3D structure models of AvrPm2 and BgtE5846, the crystal structure of BEC1054 (PDB ID:6FMB) served as a template in Phyre2 program (http://www.sbg.bio.ic.ac.uk/phyre2/html/page.cgi?id=index) and a based-on-structure prediction with AvrPm2 sequence without signal peptide was performed.

\section{Data availability statement}

All relevant data can be found within the manuscript and its supporting materials.

\section{Accession numbers}

Sequence data were deposited at the NCBI GenBank under the accession numbers MW538903 (Pm2d), MW538904 (Pm2e), MW538905 (Pm2f), MW538906 (Pm2j), MW538907 (Pm2i), MW538908 (AvrPm2H2), MW538909 (AvrPm2-H1), MW538910 (Pm2g) and MW538911 (Pm2h). All Blumeria graminis f. sp. tritici (Bgt) isolates used in this study are kept alive in the Department of Plant and Microbial Biology of the University of Zurich and are available upon request.

\section{Acknowledgements}

We thank Alexandros $\mathrm{G}$ Sotiropoulos for providing access to the genomic sequences of the Bgt isolates USA2 and USA7. We thank Patrick Ackerman and Theresa Plaschke for technical assistance. We thank Pietro Spanu for early access to the BEC1054 structure. This work was supported by the Swiss National Science Foundation (310030B_182833), the University Research Priority Program "Evolution in Action: From Genomes Ecosystems" and the Biotechnology and Biological Science Research Consil (BBSRC) funded Designing Future Wheat institute strategic program (BB/P016855/1). The authors thank the Wheat and Barley Legacy for Breeding Improvement (WHEALBI) consortium, which received funding from the European Community's Seventh Framework (F97/2007-2013) under grant agreement FP7-613556, for providing plant material and passport data.

\section{Author Contributions}

SA, BS, BBHW provided plant material and early access to Ae. tauschii sequence data. BM, HZ and JSM performed experiments. BM, CRP, AR, SA, BS, BBHW and JSM analyzed data and interpreted the results. $\mathrm{BM}, \mathrm{TK}, \mathrm{BK}$ and JSM designed the project and wrote the manuscript.

This article is protected by copyright. All rights reserved 


\section{Conflict of interest}

The authors declare that they do not have conflicting interests.

\section{Supporting information}

Figure S1 3D-model of the Pm2 LRR domain.

Figure S2 Six Pm2 variants elicit HR response upon co-infiltration with AvrPm2.

Figure S3 Inverse relationship between the intensity of HR and the number of polymorphisms of the new $\mathrm{Pm} 2$ variants compared to Pm2a upon co-infiltration with AvrPm2.

Figure S4 Variant-specific HR of Pm2i towards BgtE-5843 at different effector : NLR ratios.

Figure S5 Infection of Pm2a near isogenic lines with USA2 and USA7

Figure S6 Surface 3D models of AvrPm2 and BgtE-5846 head epitopes.

Table S1 Primers used in this study.

Table S2 Distribution of Pm2 alleles among T. aestivum and Ae. tauschii accessions used in this study.

Table S3 Protein sequence characteristics of the Pm2 variants.

Table S4 Summary of the frequency of nucleotide polymorphisms for the entire Pm2 locus among $76 T$. aestivum and Ae. tauschii accessions.

Table S5 Nucleotide polymorphism and Ka/Ks ratio of different domains of the Pm2 gene.

Table S6 Results of the two sample Wilcoxon ranks sum test.

Table S7 DNA sequences of constructs used in the AvrPm2 mutagenesis study.

Table S8 Ae. tauschii accessions used in this study.

\section{References}

Armstrong, M.R., Whisson, S.C., Pritchard, L., et al. (2005) An ancestral oomycete locus contains late blight avirulence gene Avr3a, encoding a protein that is recognized in the host cytoplasm. Proc. Natl. Acad. Sci. U. S. A., 102, 7766-71.

Arora, S., Steuernagel, B., Gaurav, K., et al. (2019) Resistance gene cloning from a wild crop relative by sequence capture and association genetics. Nat. Biotechnol., 37, 139-143.

Bauer, S., Yu, D., Lawson, A.W., et al. (2021) The leucine-rich repeats in allelic barley MLA immune 
receptors define specificity towards sequence-unrelated powdery mildew avirulence effectors with a predicted common RNase-like fold B. Kobe, ed. PLOS Pathog., 17, e1009223.

Bendahmane, A., Köhm, B.A., Dedi, C. and Baulcombe, D.C. (1995) The coat protein of potato virus X is a strain-specific elicitor of Rx1-mediated virus resistance in potato. Plant J., 8, 933-941.

Bhullar, N.K., Street, K., Mackay, M., Yahiaoui, N. and Keller, B. (2009) Unlocking wheat genetic resources for the molecular identification of previously undescribed functional alleles at the Pm3 resistance locus. Proc. Natl. Acad. Sci. U. S. A., 106, 9519-24.

Bhullar, N.K., Zhang, Z., Wicker, T. and Keller, B. (2010) Wheat gene bank accessions as a source of new alleles of the powdery mildew resistance gene Pm3: a large scale allele mining project. BMC Plant Biol., 10, 88.

Bos, J.I.B., Chaparro-Garcia, A., Quesada-Ocampo, L.M., Gardener, B.B.M. and Kamoun, S. (2009) Distinct Amino Acids of the Phytophthora infestans Effector AVR3a Condition Activation of R3a Hypersensitivity and Suppression of Cell Death. Mol. Plant-Microbe Interact., 22, 269-281.

Bos, J.I.B., Kanneganti, T.-D., Young, C., Cakir, C., Huitema, E., Win, J., Armstrong, M.R., Birch, P.R.J. and Kamoun, S. (2006) The C-terminal half of Phytophthora infestans RXLR effector AVR3a is sufficient to trigger R3a-mediated hypersensitivity and suppress INF1-induced cell death in Nicotiana benthamiana. Plant J., 48, 165-176.

Bourras, S., Kunz, L., Xue, M., et al. (2019) The AvrPm3-Pm3 effector-NLR interactions control both racespecific resistance and host-specificity of cereal mildews on wheat. Nat. Commun., 10, 2292.

Bourras, S., McNally, K.E., Ben-David, R., et al. (2015) Multiple Avirulence Loci and Allele-Specific Effector Recognition Control the Pm3 Race-Specific Resistance of Wheat to Powdery Mildew. Plant Cell, 27, 2991-3012.

Bourras, S., McNally, K.E., Müller, M.C., Wicker, T. and Keller, B. (2016) Avirulence Genes in Cereal Powdery Mildews: The Gene-for-Gene Hypothesis 2.0. Front. Plant Sci., 7, 241.

Brunner, S., Hurni, S., Streckeisen, P., Mayr, G., Albrecht, M., Yahiaoui, N. and Keller, B. (2010) Intragenic allele pyramiding combines different specificities of wheat Pm3 resistance alleles. Plant J., 64, 433-445.

Catanzariti, A.-M., Dodds, P.N., Ve, T., Kobe, B., Ellis, J.G. and Staskawicz, B.J. (2010) The AvrM Effector from Flax Rust Has a Structured C-Terminal Domain and Interacts Directly with the M Resistance Protein. Mol. Plant-Microbe Interact., 23, 49-57.

This article is protected by copyright. All rights reserved 
Chen, F., Jia, H., Zhang, X., et al. (2019) Positional cloning of PmCH1357 reveals the origin and allelic variation of the Pm2 gene for powdery mildew resistance in wheat. Crop J.

Dean, R. and Kahn, J.A.L. Van (2012) The Top 10 fungal pathogens in molecular plant pathology. Mol. Plant Pathol., 13, 414-430.

Dodds, P.N., Lawrence, G.J., Catanzariti, A.-M., Teh, T., Wang, C.-I.A., Ayliffe, M.A., Kobe, B. and Ellis, J.G. (2006) Direct protein interaction underlies gene-for-gene specificity and coevolution of the flax resistance genes and flax rust avirulence genes. Proc. Natl. Acad. Sci. U. S. A., 103, 8888-93.

Dodds, P.N. and Rathjen, J.P. (2010) Plant immunity: towards an integrated view of plant-pathogen interactions. Nat. Rev. Genet., 11, 539-548.

Dormann, C.F., Schweiger, O., Augenstein, I., et al. (2007) Effects of landscape structure and land-use intensity on similarity of plant and animal communities. Glob. Ecol. Biogeogr., 16, 774-787.

Duxbury, Z., Ma, Y., Furzer, O.J., Huh, S.U., Cevik, V., Jones, J.D.G. and Sarris, P.F. (2016) Pathogen perception by NLRs in plants and animals: Parallel worlds; Pathogen perception by NLRs in plants and animals: Parallel worlds. BioEssays, 769-781.

Ellis, J.G., Lawrence, G.J. and Dodds, P.N. (2007) Further analysis of gene-for-gene disease resistance specificity in flax. Mol. Plant Pathol., 8, 103-109.

Ellis, J.G., Lawrence, G.J., Luck, J.E. and Dodds, P.N. (1999) Identification of regions in alleles of the flax rust resistance gene $L$ that determine differences in gene-for-gene specificity. Plant Cell, 11, 495506.

Farnham, G. and Baulcombe, D.C. (2006) Artificial evolution extends the spectrum of viruses that are targeted by a disease-resistance gene from potato. Proc. Natl. Acad. Sci., 103, 18828-18833.

Franceschetti, M., Maqbool, A., Jiménez-Dalmaroni, M.J., Pennington, H.G., Kamoun, S. and Banfield, M.J. (2017) Effectors of Filamentous Plant Pathogens: Commonalities amid Diversity. Microbiol. Mol. Biol. Rev., 81, 1-17.

Glawe, D.A. (2008) The Powdery Mildews: A Review of the World's Most Familiar (Yet Poorly Known) Plant Pathogens. Annu. Rev. Phytopathol., 46, 27-51.

Guo, L., Cesari, S., Guillen, K. de, et al. (2018) Specific recognition of two MAX effectors by integrated HMA domains in plant immune receptors involves distinct binding surfaces. Proc. Natl. Acad. Sci., 115, 11637-11642.

This article is protected by copyright. All rights reserved 
Harris, C.J., Slootweg, E.J., Goverse, A. and Baulcombe, D.C. (2013) Stepwise artificial evolution of a plant disease resistance gene. Proc. Natl. Acad. Sci. U. S. A., 110, 21189-94.

Himmelbach, A., Zierold, U., Hensel, G., Riechen, J., Douchkov, D., Schweizer, P. and Kumlehn, J. (2007) A set of modular binary vectors for transformation of cereals. Plant Physiol., 145, 1192-200.

Hofgen, R. and Willmitzer, L. (1988) Storage of competent cells for Agrobacterium transformation. Nucleic Acids Res., 16.

Jones, J.D.G., Vance, R.E. and DangI, J.L. (2016) Intracellular innate immune surveillance devices in plants and animals. Science (80-. )., 35, 117-125.

Kanzaki, H., Yoshida, K., Saitoh, H., Fujisaki, K., Hirabuchi, A., Alaux, L., Fournier, E., Tharreau, D. and Terauchi, R. (2012) Arms race co-evolution of Magnaporthe oryzae AVR-Pik and rice Pik genes driven by their physical interactions. Plant J., 72, 894-907.

La Concepcion, J.C. De, Franceschetti, M., Maclean, D., Terauchi, R., Kamoun, S. and Banfield, M.J. (2019) Protein engineering expands the effector recognition profile of a rice NLR immune receptor. Elife, 8, 1-19.

Lindner, S., Keller, Bettina, Singh, S.P., Hasenkamp, Z., Jung, E., Müller, M.C., Bourras, S. and Keller, Beat (2020) Single residues in the LRR domain of the wheat PM3A immune receptor can control the strength and the spectrum of the immune response. Plant J., 104, 200-214.

Lucas, J.A., Hawkins, N.J. and Fraaije, B.A. (2015) The Evolution of Fungicide Resistance. Adv. Appl. Microbiol., 90, 29-92.

Ma, P., Xu, H., Xu, Y., et al. (2015) Molecular mapping of a new powdery mildew resistance gene Pm2b in Chinese breeding line KM2939. Theor. Appl. Genet., 128, 613-622.

Maqbool, A., Saitoh, H., Franceschetti, M., et al. (2015) Structural basis of pathogen recognition by an integrated HMA domain in a plant NLR immune receptor. Elife, 4, 213-221.

McNally, K.E., Menardo, F., Lüthi, L., et al. (2018) Distinct domains of the AVRPM3 A2/F2 avirulence protein from wheat powdery mildew are involved in immune receptor recognition and putative effector function. New Phytol., 218, 681-695.

Menardo, F., Praz, C.R., Wicker, T. and Keller, B. (2017) Rapid turnover of effectors in grass powdery mildew (Blumeria graminis). BMC Evol. Biol., 17, 223.

Müller, M.C., Praz, C.R., Sotiropoulos, A.G., et al. (2019) A chromosome-scale genome assembly reveals a 
highly dynamic effector repertoire of wheat powdery mildew. New Phytol., 221, 2176-2189.

Pace, C.N., Heinemann, U., Hahn, U. and Saenger, W. (1991) Ribonuclease T1: Structure, Function, and Stability. Angew. Chemie Int. Ed. English, 30, 343-360.

Parlange, F., Roffler, S., Menardo, F., et al. (2015) Genetic and molecular characterization of a locus involved in avirulence of Blumeria graminis f. sp. tritici on wheat Pm3 resistance alleles. Fungal Genet. Biol., 82, 181-192.

Pedersen, C., Loren van Themaat, E. Ver, McGuffin, L.J., et al. (2012) Structure and evolution of barley powdery mildew effector candidates. BMC Genomics, 13, 694.

Pennington, H.G., Gheorghe, D.M., Damerum, A., Pliego, C., Spanu, P.D., Cramer, R. and Bindschedler, L. V (2016) Interactions between the Powdery Mildew Effector BEC1054 and Barley Proteins Identify Candidate Host Targets.

Pennington, H.G., Jones, R., Kwon, S., et al. (2019) The fungal ribonuclease-like effector protein CSEP0064/BEC1054 represses plant immunity and interferes with degradation of host ribosomal RNA P. N. Dodds, ed. PLOS Pathog., 15, e1007620.

Pliego, C., Nowara, D., Bonciani, G., et al. (2013) Host-Induced Gene Silencing in Barley Powdery Mildew Reveals a Class of Ribonuclease-Like Effectors. , 26, 633-642.

Pont, C., Leroy, T., Seidel, M., et al. (2019) Tracing the ancestry of modern bread wheats. Nat. Genet., 51, 905-911.

Praz, C.R., Bourras, S., Zeng, F., et al. (2017) AvrPm2 encodes an RNase-like avirulence effector which is conserved in the two different specialized forms of wheat and rye powdery mildew fungus. New Phytol., 213, 1301-1314.

Praz, C.R., Bourras, S., Zeng, F., et al. (2019) Corrigendum to: AvrPm2 encodes an RNase-like avirulence effector which is conserved in the two different specialized forms of wheat and rye powdery mildew fungus (New Phytologist, (2017), 213, 3, (1301-1314), 10.1111/nph.14372). New Phytol., 222, 2038.

Presti, L. Lo, Lanver, D., Schweizer, G., Tanaka, S., Liang, L., Tollot, M., Zuccaro, A., Reissmann, S. and Kahmann, R. (2015) Fungal Effectors and Plant Susceptibility. Annu. Rev. Plant Biol., 66, 513-545.

Ravensdale, M., Bernoux, M., Ve, T., Kobe, B., Thrall, P.H., Ellis, J.G. and Dodds, P.N. (2012) Intramolecular Interaction Influences Binding of the Flax L5 and L6 Resistance Proteins to their AvrL567 Ligands.

This article is protected by copyright. All rights reserved 
Rodriguez-Moreno, L., Song, Y. and Thomma, B.P. (2017) Transfer and engineering of immune receptors to improve recognition capacities in crops. Curr. Opin. Plant Biol., 38, 42-49.

Sánchez-Martín, J. and Keller, B. (2019) Contribution of recent technological advances to future resistance breeding. Theor. Appl. Genet., 132, 713-732.

Sánchez-Martín, J., Steuernagel, B., Ghosh, S., et al. (2016) Rapid gene isolation in barley and wheat by mutant chromosome sequencing. Genome Biol., 17, 221-227.

Saur, I.M., Bauer, S., Kracher, B., et al. (2019) Multiple pairs of allelic MLA immune receptor-powdery mildew AVRA effectors argue for a direct recognition mechanism. Elife, $\mathbf{8}$.

Seeholzer, S., Tsuchimatsu, T., Jordan, T., et al. (2010) Diversity at the Mla Powdery Mildew Resistance Locus from Cultivated Barley Reveals Sites of Positive Selection. Mol. Plant-Microbe Interact., 23, 497-509.

Segretin, M.E., Pais, M., Franceschetti, M., Chaparro-Garcia, A., Bos, J.I.B., Banfield, M.J. and Kamoun, S. (2014) Single Amino Acid Mutations in the Potato Immune Receptor R3a Expand Response to Phytophthora Effectors. Mol. Plant-Microbe Interact., 27, 624-637.

Shen, Q., Zhou, F., Bieri, S., Haizel, T., Shirasu, K. and Schulze-lefert, P. (2003) Recognition Specificity and RAR1/SGT1 Dependence in Barley. Society, 15, 732-744.

Spanu, P.D. (2017) Cereal immunity against powdery mildews targets RNase-Like Proteins associated with Haustoria (RALPH) effectors evolved from a common ancestral gene. New Phytol., 213, 969-971.

Srichumpa, P., Brunner, S., Keller, B. and Yahiaoui, N. (2005) Allelic Series of Four Powdery Mildew Resistance Genes at the Pm3 Locus in Hexaploid Bread Wheat 1. Plant Physiol., 139, 885-895.

Stein, N., Herren, G. and Keller, B. (2001) A new DNA extraction method for high-throughput marker analysis in a large-genome species such as Triticum aestivum. Plant Breed., 120, 354-356.

Stirnweis, D., Milani, S., Jordan, T., Keller, B. and Brunner, S. (2014) Substitutions of Two Amino Acids in the Nucleotide-Binding Site Domain of a Resistance Protein Enhance the Hypersensitive Response and Enlarge the PM3F Resistance Spectrum in Wheat. Mol. Plant-Microbe Interact., 27, 265-276.

Tanksley, S.D. and Nelson, J.C. (1996) Advanced backcross QTL analysis: A method for the simultaneous discovery and transfer of valuable QTLs from unadapted germplasm into elite breeding lines. Theor. Appl. Genet., 92, 191-203.

Wang, Jizong, Hu, M., Wang, Jia, et al. (2019) Reconstitution and structure of a plant NLR resistosome 
conferring immunity. Science (80-. )., 364, eaav5870.

Wicker, T., Oberhaensli, S., Parlange, F., et al. (2013) The wheat powdery mildew genome shows the unique evolution of an obligate biotroph. Nat. Genet., 45, 1092-1096.

Xu, H., Yi, Y., Ma, P., Qie, Y., Fu, X., Xu, Y., Zhang, X. and An, D. (2015) Molecular tagging of a new broadspectrum powdery mildew resistance allele Pm2c in Chinese wheat landrace Niaomai. Theor. Appl. Genet., 128, 2077-2084.

Yahiaoui, N., Srichumpa, P., Dudler, R. and Keller, B. (2004) Genome analysis at different ploidy levels allows cloning of the powdery mildew resistance gene Pm3bfrom hexaploid wheat. Plant J., 37, 528538. 


\section{Figure 1 Protein sequence comparison of the Pm2 protein variants.}

Schematic representation of the Pm2 protein with the predicted domains: CC domain (black), the NB-ARC domain (blue) and the LRR domain (red). Amino acid positions are indicated vertically from top to bottom (e.g. the last position is number 1230), positions 170, 398, and 1167 are bold. The color of the numbers corresponds to the domains, where the polymorphisms occur. The top row corresponds to the amino acids in Pm2a. Amino acid changes occurring in the Pm2 proteins compared to the Pm2a protein are indicated. Dots represent identical amino acids compared to Pm2a, whereas dashes represent amino acid deletions. LRR 21 is indicated with the two corresponding amino acid polymorphisms at positions 1167 and 1169.

Figure 2 Pm2 variant specific-HR upon co-infiltration with AvrPm2 and BgtE-5843.

Quantification of the HR at 2 days post-infiltration (dpi) of Pm2 variants with AvrPm2 (a), or BgtE-5843 (b). Red and black data points refer to two independent experiments with 7-8 replicates each. Statistical significance was assessed with a two-sided Wilcoxon rank-sum test for unpaired data and p-values are indicated (n. s. = not significant). Mean values are indicated by the middle line in the boxplot. (c) Representative pictures of the HR in co-infiltrated $\mathrm{N}$. benthamiana leaves. (d) N-terminal HA-tagged proteins were used for western blots, except for a C-terminal HA-tagged AvrPm2, since the N-terminal tagged variant was not detectable. Western blot probed with anti-HA antibodies show protein accumulation. Expected protein size for Pm2 variants and effectors is at $143 \mathrm{kDa}$ and $13 \mathrm{kDa}$, respectively. Ponceau staining of the western blot membrane (lower panel) is depicted.

Figure $3 \mathrm{Pm}^{1167}$ is responsible for the variant-specific HR of Pm2a and Pm2i towards AvrPm2 and BgtE5843 , respectively.

Quantification of the HR at 2 dpi after co-infiltration of the Pm2 mutants with AvrPm2 (a), or BgtE-5843 (b). Red and black data points refer to two independent experiments with 7-8 replicates each. Statistical significance was assessed with a two-sided Wilcoxon rank-sum test for unpaired data and p-values are indicated (n. s. = not significant). Mean values are indicated by the middle line in the boxplot. (c) Schematic representation of Pm2a mutants at positions 170, 398 and 1167. (d) Section of the 3D model of the Pm2a LRR domain. Glycine at position 1167 is indicated in red. (e) Section of the 3D model of the Pm2i LRR domain. Aspartic acid at position 1167 is indicated in red.

Figure 4 Pm2a triggers HR upon co-infiltration with AvrPm2-H1 variant.

(a) Alignment of AvrPm2, AvrPm2-H1 and AvrPm2-H2 amino acid sequences. Dots represent identical amino acids compared to AvrPm2. (b) Quantification of the HR induced upon co-infiltration of Pm2a with 
the AvrPm2 variants and E103 (AvrPm2 (V94D)) at 2 dpi. Red and black data points refer to two independent experiments with 7-8 replicates each. Statistical significance was assessed with a two-sided Wilcoxon rank sum test for unpaired data and $p$-values are indicated (n. s. = not significant). Mean values are indicated by the middle line in the boxplot. (c) Western blot probed with anti-HA antibodies show protein accumulation at the expected size of $13 \mathrm{kDa}$. Ponceau staining of the western blot membrane (lower panel) is depicted.

Figure 5 Protein sequence alignment and 3D structure comparison of AvrPm2, BgtE-5843 and BgtE5846.

(a) Amino acid sequence alignment of AvrPm2, BgtE-5843 and BgtE-5846. Top row indicates amino acid position, bottom row indicates units \#1- \#8. (b) Superposition of secondary structure 3D model of AvrPm2 (green) and BgtE-5846 (blue) based on the protein structure of BEC1054 (99.4\% and 99\% confidence, respectively). Since BEC1054 is one amino acid shorter than AvrPm2 and the last amino acid is missing in the protein structure, the last two amino acids of AvrPm2 and the last amino acid of BgtE-5846 are not included in the 3D model. (c) 3D model of AvrPm2. Amino acids of the same colour represent units which were swapped between AvrPm2 and BgtE-5846 and correspond to the colours in (a).

Figure 6 Functional analysis of the Pm2a interaction with effector mutants and wild type AvrPm2 and BgtE-5846 in N. benthamiana.

(a) Schematic representation of effector mutants. Green colour indicates amino acid sequence of AvrPm2, blue colour indicates amino acid sequence of BgtE5846. HR intensity refers to co-infiltration with Pm2a.(b, d) Quantification of the HR two days after co-infiltration of Pm2a together with AvrPm2, BgtE-5846 and mutants in the $N$. benthamiana assay, respectively. Red and black data points refer to two independent experiments with 7-8 replicates each. Statistical significance was assessed with a two-sided Wilcoxon rank-sum test for unpaired data and $p$-values are indicated (n. s. = not significant). Mean values are indicated by the middle line in the boxplot. (c) Western blot probed with anti-HA antibodies show protein accumulation. Effectors are visible at expected protein size of $13 \mathrm{kDa}$. Ponceau staining of the western blot membrane (lower panel) is depicted.

\section{Figure 7 Functional analysis of effector mutants with Pm2 variants in N. benthamiana.}

(a) Schematic representation of AvrPm2 (green), BgtE-5846 (blue), BgtE-5843 (red), E33 and E98. HR intensity refers to co-infiltration with Pm2a. (b, c) Quantification of the HR induced upon co-infiltration in N. benthamiana of Pm2 variants with construct E33 (b), or construct E98 (c) at 2 dpi. Red and black data points refer to two independent experiments with 7-8 replicates each. Statistical significance was 
assessed with a two-sided Wilcoxon rank-sum test for unpaired data and p-values are indicated (n. s. = not significant). Mean values are indicated by the middle line in the boxplot.

The following Supporting Information is available for this article:

\section{Figure S1 3D-model of the Pm2 LRR domain.}

3D-model of the Pm2 LRR domain. LRR21 (1163-1186) is marked in yellow. The solvent exposed polymorphic amino acid at position 1167 is marked in red. LRR domain in side view (left) and 70 clockwise rotated (right).

Figure S2 Six Pm2 variants elicit HR response upon co-infiltration with AvrPm2.

Quantification of the HR induced upon co-infiltration of AvrPm2 family members with the Pm2 variants at $2 \mathrm{dpi}$. Red and black data points refer to two independent experiments with 7-8 replicates each. Statistical significance was assessed with a two-sided Wilcoxon rank sum test for unpaired data and $p$-values are indicated (n. s. = not significant). Mean values are indicated by the middle line in the boxplot.

Figure S3 Inverse relationship between the intensity of HR and the number of polymorphisms of the new Pm2 variants compared to Pm2a upon co-infiltration with AvrPm2.

Quantification of the HR at $2 \mathrm{dpi}$ of Pm2 variants with AvrPm2. Numbers in brackets refer to the amino acid changes of the Pm2 variants compared to Pm2a. Red and black data points refer to two independent experiments with 7-8 replicates each. Statistical significance was assessed with a two-sided Wilcoxon rank sum test for unpaired data and p-values are indicated (n. s. = not significant). Mean values are indicated by the middle line in the boxplot.

Figure S4 Variant-specific HR of Pm2i towards BgtE-5843 at different effector : NLR ratios.

Quantification of the HR induced upon co-infiltration of BgtE-5843 members with Pm2a or Pm2i at 2 dpi in the effector: NLR ratios $4: 1,1: 1$ and $1: 4$. Red and black data points refer to two independent experiments with 7-8 replicates each. Statistical significance was assessed with a two-sided Wilcoxon rank sum test for unpaired data and p-values are indicated (n. s. = not significant). Mean values are indicated by the middle line in the boxplot.

\section{Figure S5 Infection of Pm2a near isogenic lines with USA2 and USA7}

Leaf segment infection test with the Bgt isolates USA2 and USA7 on the Pm2a near isogenic wheat lines Ulka/8*Chancellor, Federation*4/Ulka and $\mathrm{Cl} 12632 / 8 *$ Chancellor. 
Figure S6 Surface 3D models of AvrPm2 and BgtE-5846 head epitopes.

(a) Front view of AvrPm2 head epitope (green). The eight amino acids which differ from the ones in the BgtE-5846 head epitope are marked in red. (b) Side view of AvrPm2 head epitope. (c) Front view of BgtE5846 head epitope (blue). The amino acids, which differ from the ones in the AvrPm2 head epitope are marked in red. (d) Side view of BgtE-5846 head epitope.

This article is protected by copyright. All rights reserved 
LRR 21

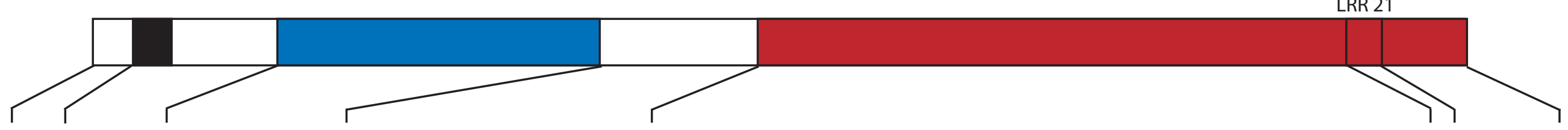

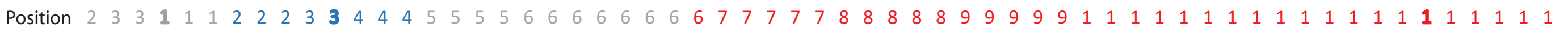

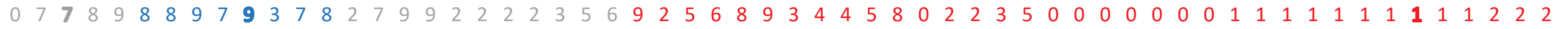
$\begin{array}{lllllllllllllllllllllllllllllllllllllllllllllllllllllllllll}0 & 2 & 1 & 3 & 4 & 8 & 8 & 8 & 2 & 1 & 6 & 7 & 0 & 7 & 8 & 2 & 3 & 8 & 9 & 1 & 9 & 0 & 6 & 8 & 9 & 3 & 4 & 7 & 4 & 0 & 3 & 7 & 3 & 0 & 7 & 9 & 2 & 7 & 0 & 0 & 1 & 2 & 3 & 5 & 6 & 1 & 1 & 2 & 3 & 4 & 4 & 4 & 6 & 6 & 9 & 1 & 1 & 3\end{array}$

$\begin{array}{llllllllllllllllllll}3 & 7 & 6 & 2 & 0 & 8 & 0 & 7 & 9 & 0 & 9 & 1 & 3 & 4 & 7 & 9 & 1 & 2 & 4 & 0\end{array}$



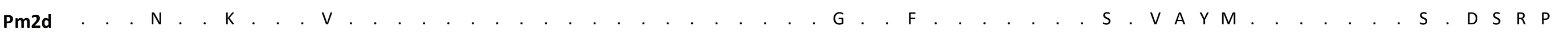

Pm2e

Pm2f

$$
E \cdot R \quad R \cdot R \cdot \cdot Q
$$

Pm2g

$$
E \cdot R R \cdot R V . . .-S A C V . P I . . N . G K M F
$$

$S \cdot V A Y M$

D $S$ R P

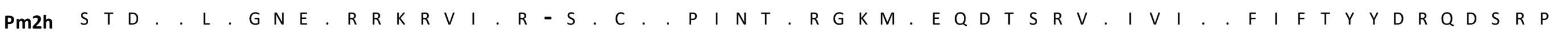

Pm2i $\mathrm{N} \cdot \cdot \cdot \cdot \cdot \cdot \mathrm{V}$

Pm2j N 
(a)



(c)



BgtE-5843

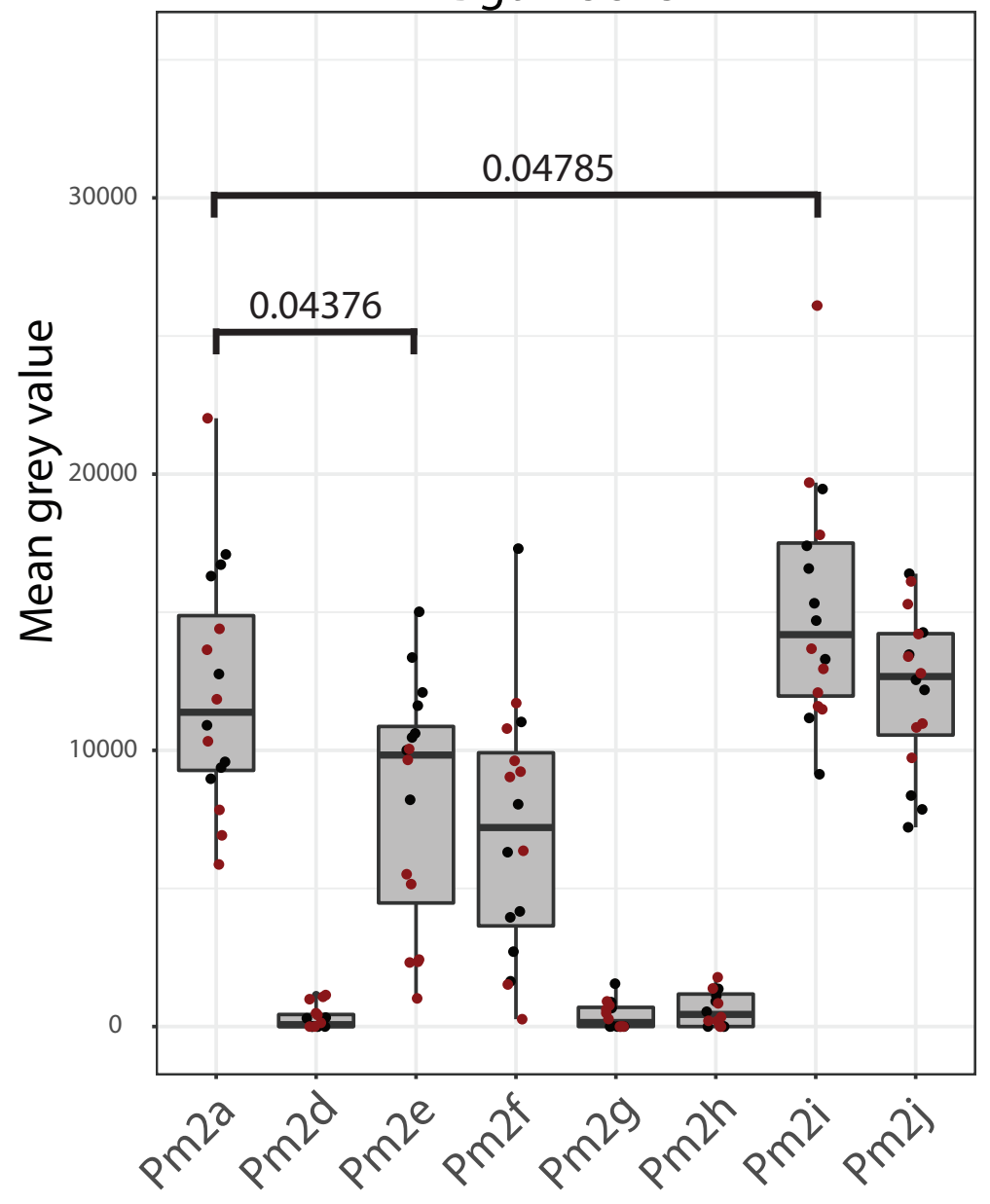


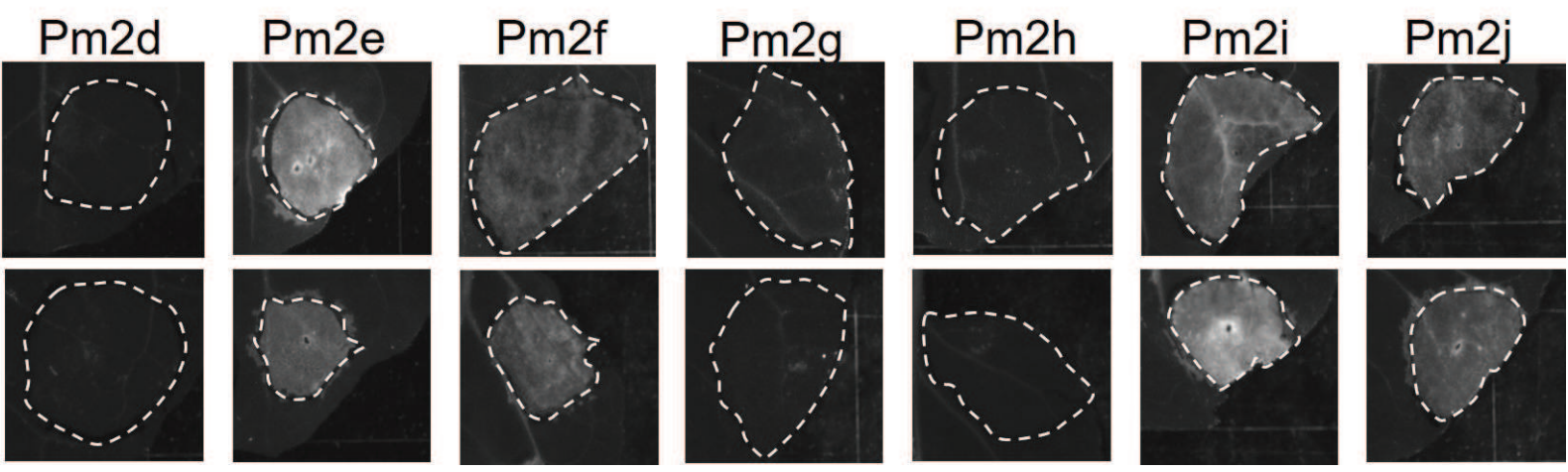

(d)
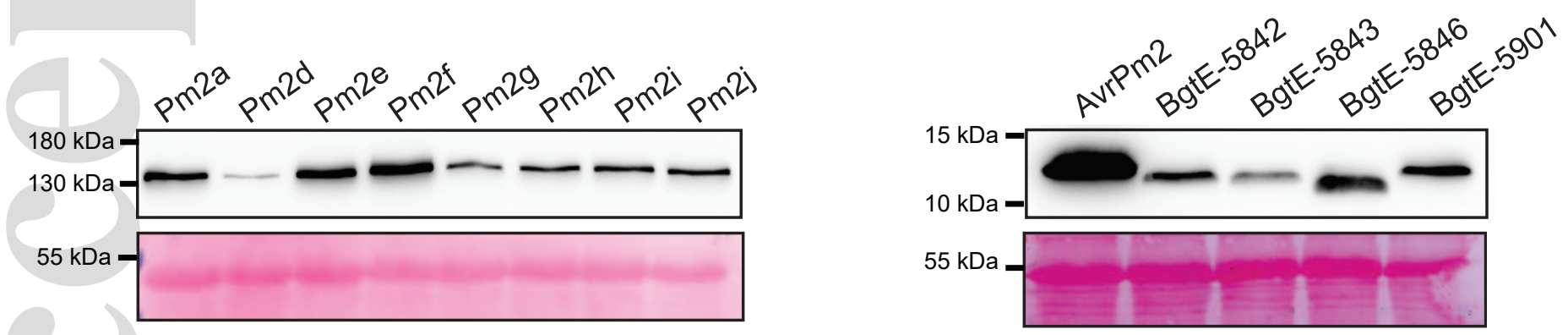
(a)

AvrPm2 tpj_15214_f3.pd(b)

BgtE-5843

4.29E-05



$b^{20} r^{s^{100}} a^{n^{2}}$

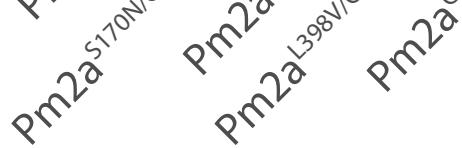

(c)
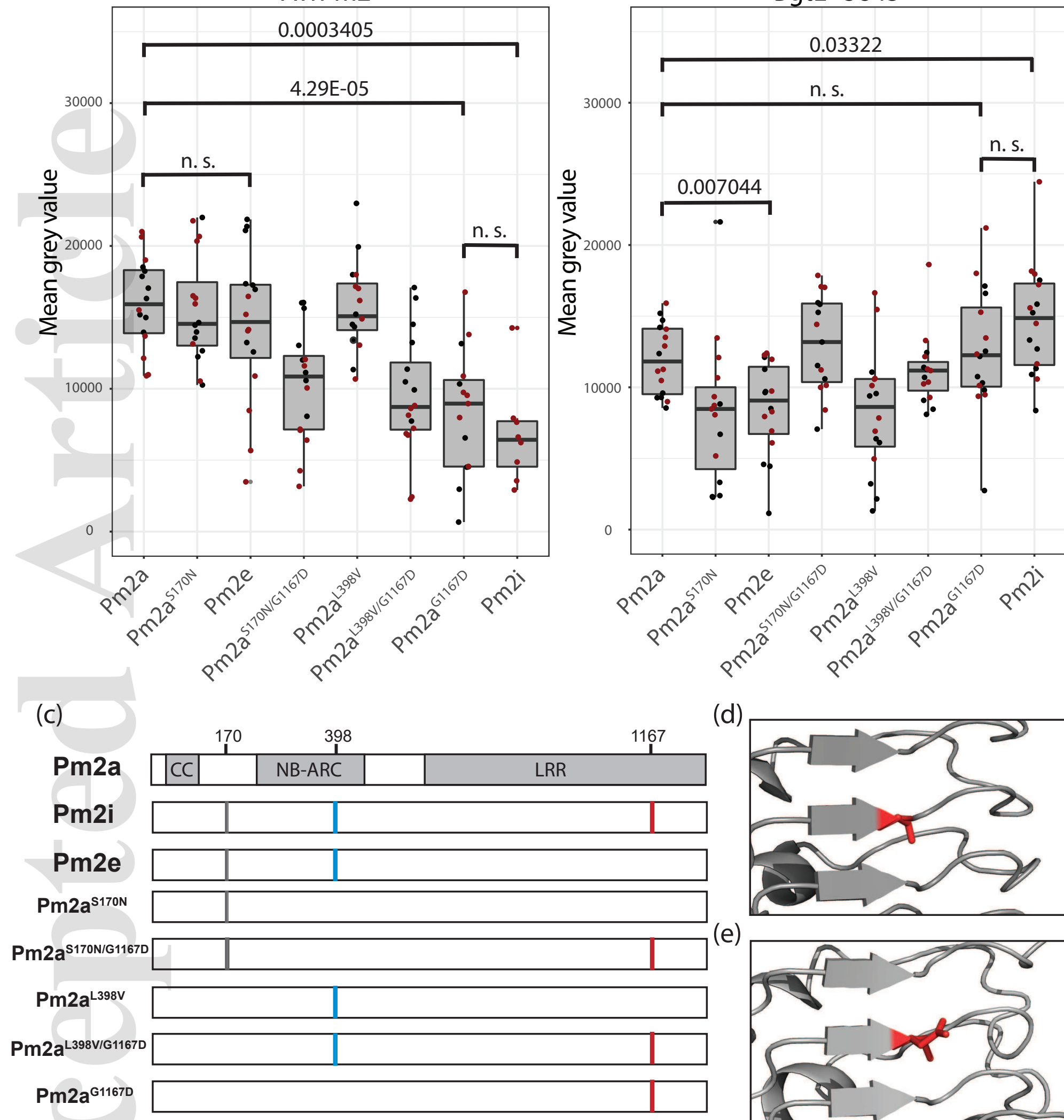

c)

${ }^{170}$

Pm2as170N/G1167

Pm2a ${ }^{\text {L398V }}$

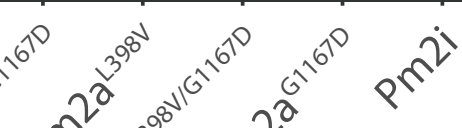


AvrPm2

MESYWDCKGIPILFRTVHAAVELAFTSQPGSISGYPSICRTTPLRTGPDERRQFPLTDTGARWQGGGITYYVEATRDKRHCEVFGTAGGVYKCTLVLRD

AvrPm2-H1

AvrPm2-H2

(b)



(c)

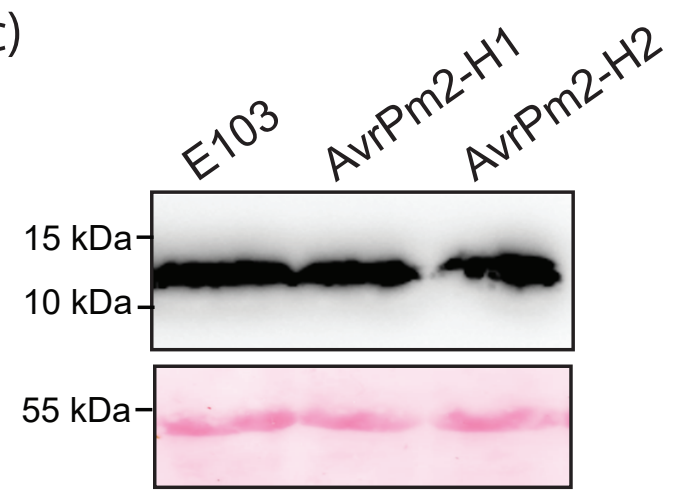

This article is protected by copyright. All rights reserved 
(a)

AvrPm2 BgtE-5843

BgtE-5846



$27-36 \quad 37-39 \quad 40-51 \quad 52-55$

56-70

71-74 75-80 81-84 85-92

93-99

(b)

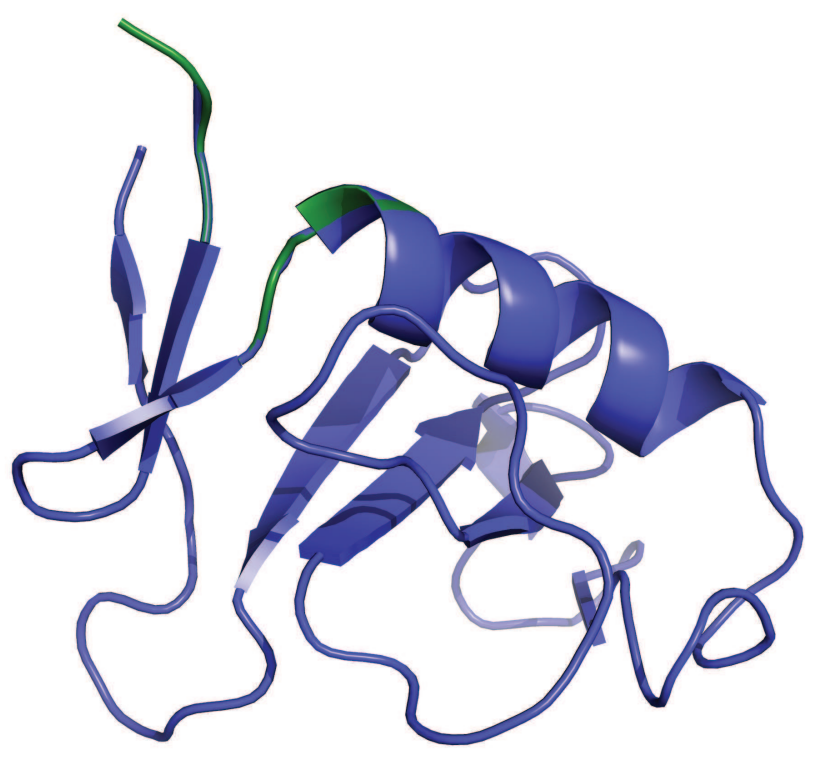

(c)



This article is protected by copyright. All rights reserved 
(a)



(b)

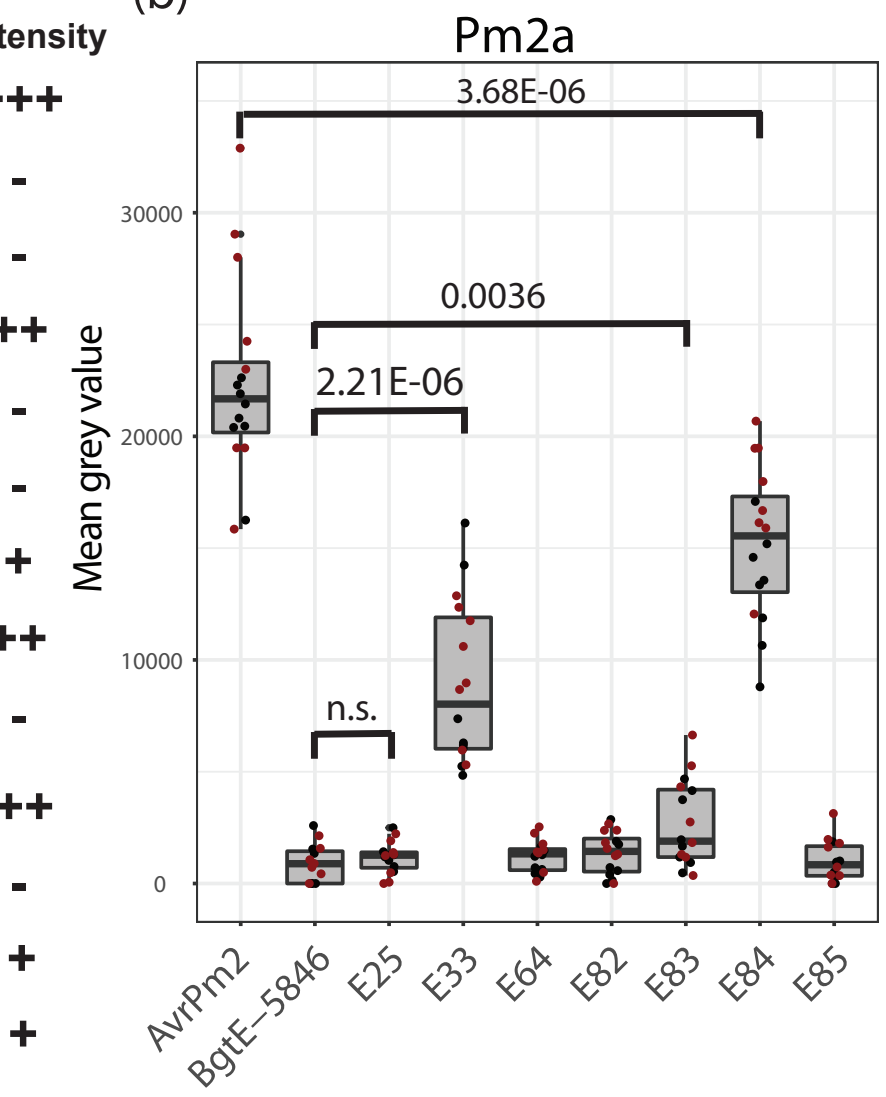


(a)

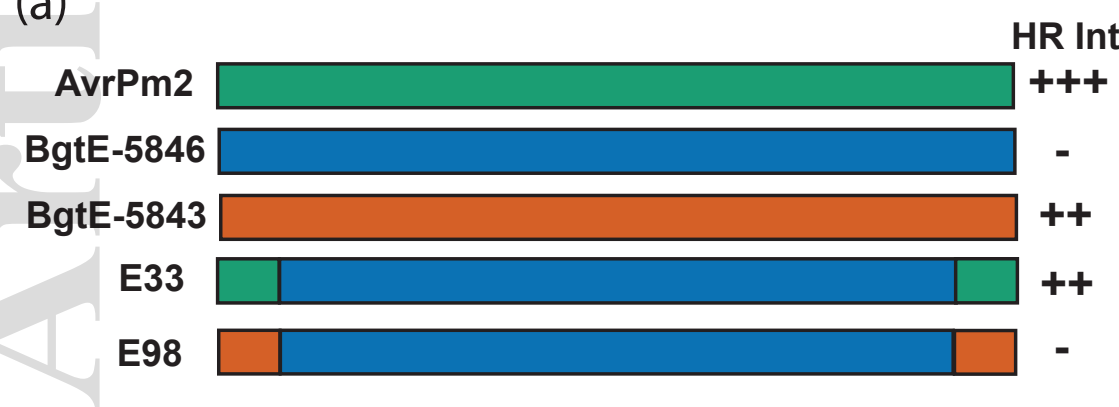

(b)

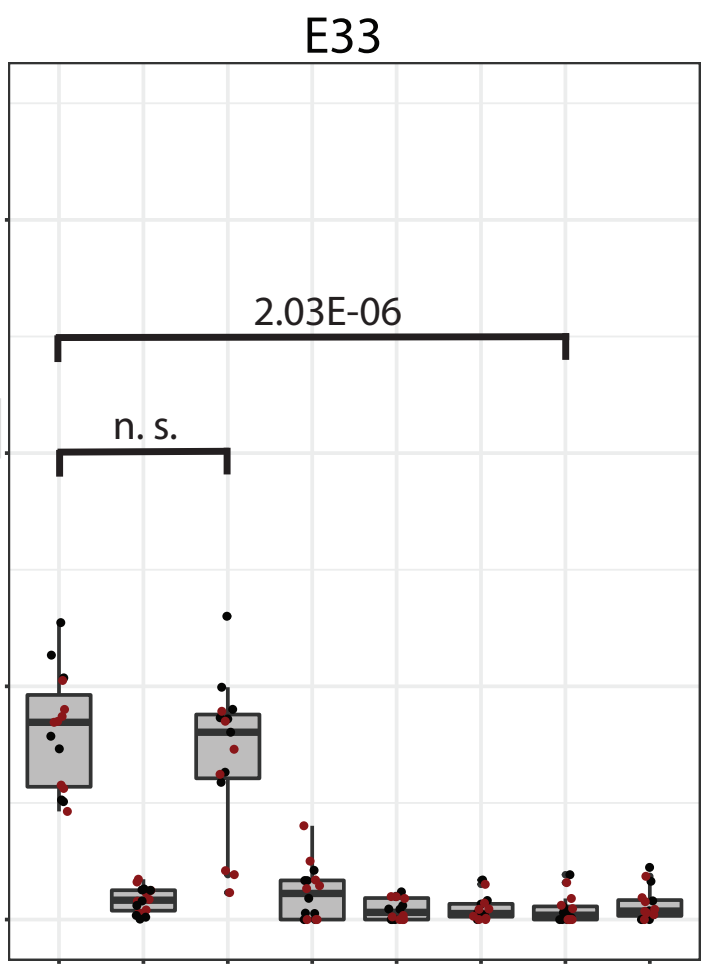

(c)

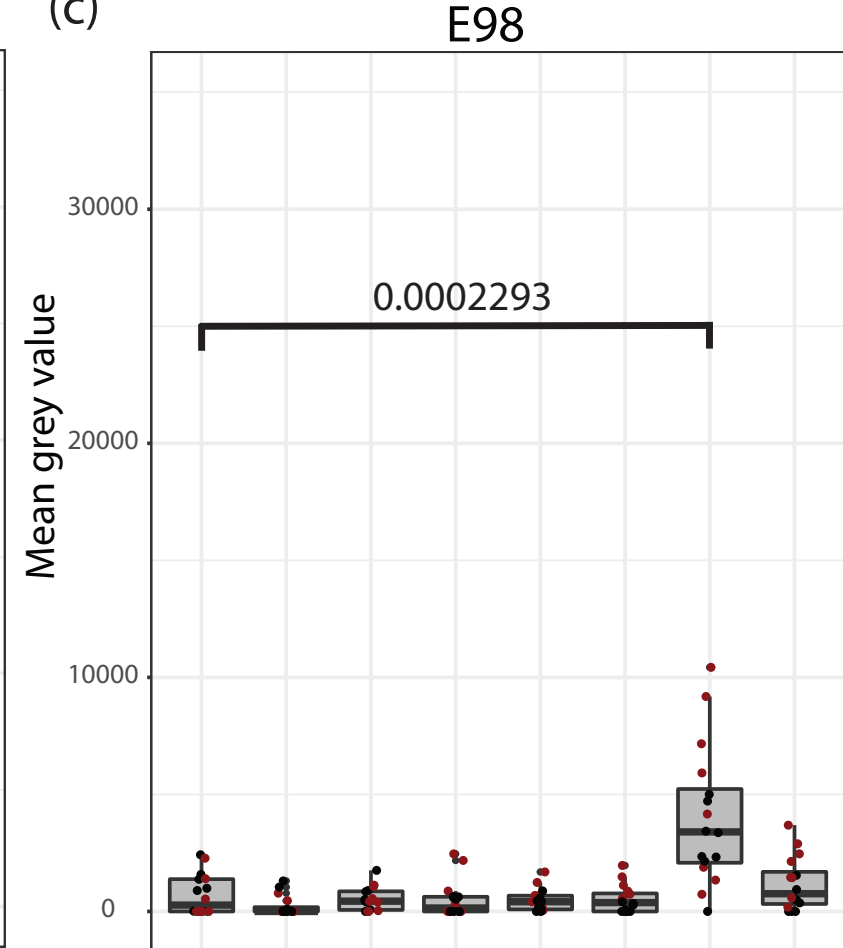

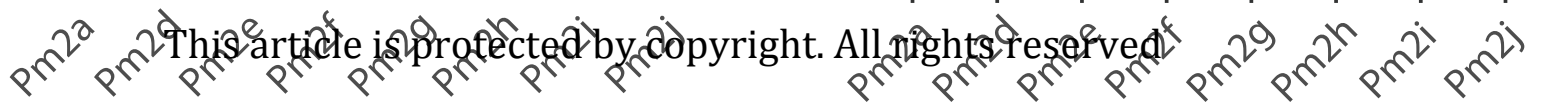

